\title{
HIGHLY SELECTIVE DEBLOCKING OF PROPARGYL CARBONATES \\ IN THE PRESENCE OF PROPARGYL CARBAMATES WITH
}

\section{TETRATHIOMOLYBDATE}

\author{
Ramesh R, Ramakrishna G Bhat, Srinivasan Chandrasekaran \\ Department of Organic Chemistry, Indian Institute of Science
}

Bangalore-560 012, India

Table of Contents

\begin{tabular}{|l|l|}
\hline Experimental Procedures & Page numbers S2-S12 \\
\hline${ }^{1} \mathrm{H}$ and ${ }^{13} \mathrm{C}$ nmr spectra for compounds 4a-h & Page numbers S13-S20 \\
\hline${ }^{1} \mathrm{H}$ and ${ }^{13} \mathrm{C}$ nmr spectra for compounds 7a-h & Page numbers S21-S28 \\
\hline${ }^{1} \mathrm{H}$ and ${ }^{13} \mathrm{C}$ nmr spectra for compounds 8a-h & Page numbers S29-S36 \\
\hline
\end{tabular}




\section{Experimental Procedures}

All reactions were performed in oven dry apparatus and were stirred magnetically. Melting points reported are uncorrected. Ultrasonication was carried out using an ultrasonic cleaning bath of $40 \mathrm{KHz}$ frequency. Infrared spectra were recorded using an FT-IR instrument and the frequencies are reported in wave number $\left(\mathrm{cm}^{-1}\right)$ and intensities of the peaks are denoted as $\mathrm{s}$ (strong), w (weak), m (medium) and br (broad). ${ }^{1} \mathrm{H}$ and ${ }^{13} \mathrm{C}$ NMR spectra were recorded on a 300 $\mathrm{MHz}$ and $75 \mathrm{MHz}$ spectrometer respectively. Chemical shifts are reported in parts per million downfield from the internal reference, tetramethylsilane. Multiplicity is indicated using the following abbreviations: s (singlet), d (doublet), $\mathrm{t}$ (triplet), q (quartet), qi (quintet), m (multiplet), sb (broad singlet), db (broad doublet). Coupling constants are reported wherever it is necessary in Hertz (Hz). Mass spectra were recorded on a Q-TOF electrospray instrument.

\section{Preparation of Propargyloxycarbonyl chloride (Poc-Cl) 1}

To a stirred solution of triphosgene $(2.23 \mathrm{~g}, 7.5 \mathrm{mmol})$ in dry ether $(30 \mathrm{~mL})$, activated charcoal $(0.05 \mathrm{~g})$ was added and stirred overnight at room temperature $\left(28^{\circ} \mathrm{C}\right)$. The solution was cooled to $0{ }^{\circ} \mathrm{C}$ and propargyl alcohol $(0.9 \mathrm{~mL}, 15 \mathrm{mmol})$ in dry ether $(10 \mathrm{~mL})$ was added drop wise. The resultant solution was stirred for 12 hours and filtered. The ethereal layer was distilled under reduced pressure and the concentrated liquid was used for reactions without any further purification.

${ }^{1} \mathrm{H}$ NMR: $2.6(\mathrm{t}, 1 \mathrm{H}, J=2.4), 4.7(\mathrm{~d}, 2 \mathrm{H}, J=2.4) .{ }^{13} \mathrm{C} \mathrm{NMR}: 150.2,77.6,74.9,58.3 . \mathrm{IR}$ (neat): 3303, 2982, 2131, 1777.

\section{Preparation of ammonium tetrathiomolybdate:}

Ammonium molybdate (10 g) was dissolved in a mixture of ammonium hydroxide $(60 \mathrm{~mL})$ water $(20 \mathrm{~mL})$, and the solution was filtered. Hydrogen sulfide was bubbled rapidly at ambient temperature into the solution mixture until it was saturated and then the temperature was raised to $60{ }^{\circ} \mathrm{C}$ while maintaining slow stream if hydrogen sulfide. After $60 \mathrm{~min}$, the mixture was 
cooled to $0{ }^{\circ} \mathrm{C}$ for $30 \mathrm{~min}$ and the product was isolated by filtration. The crystalline solid was washed with isopropyl alcohol $(25 \mathrm{ml})$, ether $(25 \mathrm{ml})$ and dried under vacuum to obtained brick red crystals of ammonium molybdate (13.4- $14.2 \mathrm{~g}, 92 \%)$.

UV-Vis $\left(\mathrm{H}_{2} \mathrm{O}\right): \lambda_{\max }(\square) 467(11,850), 316(16,750), 241(24,700)$. IR (KBr): 750, 700, 525, 450

\section{Preparation of Benzyltriethylammonium tetrathiomolybdate 2}

A solution of benzyltriethylammonium chloride $(23.31 \mathrm{~g}, 102.5 \mathrm{mmol})$ in distilled water $(60 \mathrm{~mL})$ was added to rapidly stirred solution of ammonium tetrathiomolybdate $(13 \mathrm{~g}, 50 \mathrm{~mL})$ in distilled water $(60 \mathrm{~mL})$. Rapid stirring was stirring was continued for $2 \mathrm{~h}$ at room temperature and the solid that separated was filtered, washed with isopropyl alcohol (40 ml) and diethylether (40 ml). After drying in high vacuum, brick red powder of benzyltriethylammonium tetrathiomolybdate (2) was stored in a desiccator $(24 \mathrm{~g}, 80 \%)$.

mp: $150{ }^{\circ} \mathrm{C}$ (decomp) . UV-Vis (DMF): $\lambda_{\max }(\square) 472(11,850) ; 320(16,750) ; 274(24,700)$.

\section{General procedure for the protection of amines, alcohols and phenols with $\mathrm{PocCl}, 1$}

To a well-stirred solution of the amine, alcohol, or phenol $(10 \mathrm{mmol})$ and triethylamine $(10.5$ $\mathrm{mmol})$ in anhydrous dichloromethane $(30 \mathrm{~mL})$, at the specified temperature (Table 1), PocCl (10 mmol) was added dropwise. The reaction mixture was stirred at the same temperature till the completion of the reaction (as shown by TLC). It was then washed with water and dried over anhydrous $\mathrm{Na}_{2} \mathrm{SO}_{4}$. The carbamate, or carbonate was then purified using flash column chromatography on a silica gel (230-400 mesh) column using ethylacetate/hexane mixtures of appropriate composition for elution.

\section{Compound 4a:}

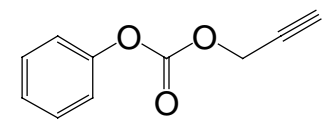

Pale yellow oil. ${ }^{1} \mathrm{H}$ NMR $\left(\mathrm{CDCl}_{3}\right): \delta$ 7.37-7.15 (m, 5H), $4.8(\mathrm{~d}, \mathrm{~J}=2.7,2 \mathrm{H}), 2.6(\mathrm{t}, \mathrm{J}=2.7,1 \mathrm{H})$.

${ }^{13} \mathrm{C} \mathrm{NMR}\left(\mathrm{CDCl}_{3}\right): \delta 152.8,150.7,129.3,126.0,120.7,76.4,76.1,55.6$. IR (neat): 3293 (s), 
3069 (w), 2130 (w), 1759 (s). ESMS: HRMS calculated for $\mathrm{C}_{10} \mathrm{H}_{8} \mathrm{O}_{3}+\mathrm{Na}$ : 199.0371, found: 199.0390.

\section{Compound 4b:}

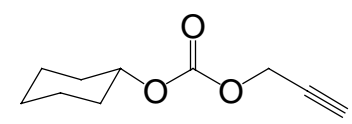

Colorless oil. ${ }^{1} \mathrm{H}$ NMR $\left(\mathrm{CDCl}_{3}\right): \delta 4.7(\mathrm{~d}, \mathrm{~J}=2.4,2 \mathrm{H}), 4.6(\mathrm{qi}, \mathrm{J}=4.2,1 \mathrm{H}), 2.5(\mathrm{t}, \mathrm{J}=2.4,1 \mathrm{H})$, 2.1-1.2 (m, 10H). ${ }^{13} \mathrm{C}$ NMR: $\delta 153.8,77.2,77.1,75.3,54.7,31.3,25.0,23.4$. IR (neat): $2130(\mathrm{w})$, 1747 (s). ESMS: HRMS calculated for $\mathrm{C}_{10} \mathrm{H}_{14} \mathrm{O}_{3}+\mathrm{Na}$ : 205.0841, found: 205.0849.

\section{Compound 4c:}

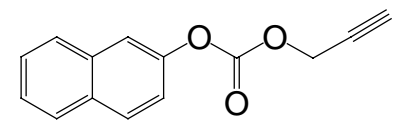

White solid, mp: $70{ }^{\circ} \mathrm{C} .{ }^{1} \mathrm{H} \mathrm{NMR}\left(\mathrm{CDCl}_{3}\right): \delta 7.8(\mathrm{~m}, 3 \mathrm{H}), 7.7(\mathrm{~d}, \mathrm{~J}=2.1,1 \mathrm{H}), 7.5-7.4(\mathrm{~m}, 2 \mathrm{H})$, $7.3(\mathrm{dd}, \mathrm{J}=4.35, \mathrm{~J}=2.7,1 \mathrm{H}), 4.8(\mathrm{~d}, \mathrm{~J}=2.7,2 \mathrm{H}), 2.6(\mathrm{t}, \mathrm{J}=2.7,1 \mathrm{H}) .{ }^{13} \mathrm{C} \mathrm{NMR}\left(\mathrm{CDCl}_{3}\right): \delta 153.1$, $148.5,133.5,131.5,129.6,127.74,127.72,126.7,125.9,120.2,117.9,55.9 . \mathrm{IR}(\mathrm{KBr}): 3289$ (s), 2134 (w), 1769 (s). ESMS: HRMS calculated for $\mathrm{C}_{14} \mathrm{H}_{10} \mathrm{O}_{3}+\mathrm{Na}$ : 249.0528, found: 249.0523.

\section{Compound 4d:}<smiles>C#CCOC(=O)OC=CCC</smiles>

Colorless oil. ${ }^{1} \mathrm{H}$ NMR $\left(\mathrm{CDCl}_{3}\right): \delta 4.7(\mathrm{~d}, \mathrm{~J}=2.7,2 \mathrm{H}), 4.2(\mathrm{t}, \mathrm{J}=6.6,2 \mathrm{H}), 2.5(\mathrm{t}, \mathrm{J}=2.7,1 \mathrm{H}), 1.7-$ $1.6(\mathrm{~m}, 2 \mathrm{H}), 1.3(\mathrm{sb}, 18 \mathrm{H}), 0.88(\mathrm{t}, \mathrm{J}=6.9,3 \mathrm{H}) .{ }^{13} \mathrm{C} \mathrm{NMR}\left(\mathrm{CDCl}_{3}\right): \delta 154.6,75.4,68.6,54.9$, 29.6, 29.5, 29.4, 29.4, 29.2, 29.2, 29.1, 28.5, 25.6, 22.6, 14.0. IR (neat): 2923 (s), 2123.3 (w), 1748 (s). ESMS: HRMS calculated for $\mathrm{C}_{16} \mathrm{H}_{28} \mathrm{O}_{3}+\mathrm{Na}$ : 291.1936, found: 291.1946.

\section{Compound 4e:}

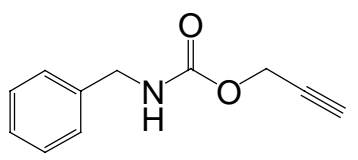


White solid, mp: $52{ }^{\circ} \mathrm{C} .{ }^{1} \mathrm{H}$ NMR $\left(\mathrm{CDCl}_{3}\right): \delta$ 7.28-7.32 (m, 5H), $5.2(\mathrm{sb}, 1 \mathrm{H}), 4.7(\mathrm{~s}, 2 \mathrm{H}), 4.4$ $(\mathrm{d}, \mathrm{J}=5.4,2 \mathrm{H}), 2.5(\mathrm{~s}, 1 \mathrm{H}) .{ }^{13} \mathrm{C} \mathrm{NMR}\left(\mathrm{CDCl}_{3}\right): \delta 155.4,138.0,128.6,127.5,127.4,78.2,74.6$, 52.5, 45.1. IR (thin film): 3313 (s), 3030 (w), 2129 (w), 1690 (s). ESMS: HRMS calculated for $\mathrm{C}_{11} \mathrm{H}_{11} \mathrm{NO}_{2}+\mathrm{Na}: 212.0687$, found: 212.0692 .

\section{Compound 4f:}

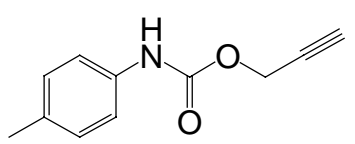

White solid, mp: $98{ }^{\circ} \mathrm{C} .{ }^{1} \mathrm{H}$ NMR $\left(\mathrm{CDCl}_{3}\right): \delta 7.3(\mathrm{~d}, \mathrm{~J}=8.4,2 \mathrm{H}), 7.110(\mathrm{~d}, \mathrm{~J}=8.4 \mathrm{~Hz}, 2 \mathrm{H}), 6.7$ $(\mathrm{sb}, 1 \mathrm{H}), 4.8(\mathrm{~d}, \mathrm{~J}=2.1 \mathrm{~Hz}, 2 \mathrm{H}), 2.5(\mathrm{t}, \mathrm{J}=2.1 \mathrm{~Hz}, 1 \mathrm{H}), 2.3(\mathrm{~s}, 3 \mathrm{H}) .13 \mathrm{C} \mathrm{NMR}\left(\mathrm{CDCl}_{3}\right): \delta 152.5$ 134.7, 133.4, 129. 6, 118.8, 78.5, 74.9, 52.6, 20.7. IR (KBr): 3314 (s), 3030 (w), 2132 (w), 1698 (s). ESMS: HRMS calculated for $\mathrm{C}_{11} \mathrm{H}_{11} \mathrm{NO}_{2}+\mathrm{Na}$ : 212.0687, found: 212.0694.

\section{Compound 4g:}

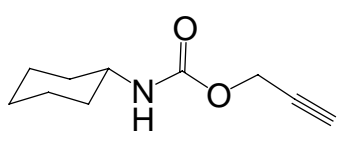

White solid, mp: $58{ }^{\circ} \mathrm{C} .{ }^{1} \mathrm{H}$ NMR $\left(\mathrm{CD}_{3} \mathrm{OD}\right): \delta 4.6$ (s, 2H), 3.29-3.37 (m, $\left.1 \mathrm{H}\right), 2.8(\mathrm{~s}, 1 \mathrm{H})$, 1.13-1.87 (m, $10 \mathrm{H}) .{ }^{13} \mathrm{C}$ NMR $\left(\mathrm{CD}_{3} \mathrm{OD}\right): \delta 157.1,79.6,75.6,52.8,51.4,26.6,26.1$. IR (thin film): 3289 (br), 2931 (s), 2122 (w), 1707 (s). ESMS: HRMS calculated for $\mathrm{C}_{10} \mathrm{H}_{15} \mathrm{NO}_{2}+\mathrm{Na}$ : 204.1000, found: 204.1010 .

Compound 4h:

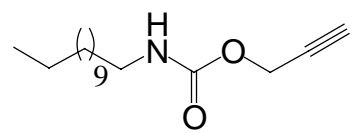

White solid, mp: $54{ }^{\circ} \mathrm{C} .{ }^{1} \mathrm{H}$ NMR $\left(\mathrm{CDCl}_{3}\right): \delta 4.8(\mathrm{sb}, 1 \mathrm{H}), 4.6(\mathrm{~d}, \mathrm{~J}=1.5,2 \mathrm{H}), 3.2(\mathrm{q}, \mathrm{J}=6.6,2 \mathrm{H})$, $2.5(\mathrm{t}, \mathrm{J}=1.6,1 \mathrm{H}), 1.5(\mathrm{t}, \mathrm{J}=6.6,2 \mathrm{H}), 1.3(\mathrm{sb}, 18 \mathrm{H}), 0.88(\mathrm{t}, \mathrm{J}=6.6,3 \mathrm{H}) .{ }^{13} \mathrm{C} \mathrm{NMR}\left(\mathrm{CDCl}_{3}\right): \delta$ $155.4,78.3,74.4,52.3,41.1,31.9,29.8,29.58,29.52,29.3,29.2,26.7,22.6,14.1$. IR (thin film): 
3300 (s), 2920 (s), 2852 (s), 2123 (w), 1715 (s). ESMS: HRMS calculated for $\mathrm{C}_{16} \mathrm{H}_{29} \mathrm{NO}_{2}+\mathrm{Na}$ : 290.2096, found: 290.2096.

Procedure for the selective deblocking of propargyl carbonates in presence of propargyl carbamates

To a solution of propargyl carbonate and carbamate $\left(2 \mathrm{mmol}\right.$ each) in anhydrous $\mathrm{CH}_{3} \mathrm{CN}(2 \mathrm{~mL})$, tetrathiomolybdate $2(2.2 \mathrm{mmol})$ was added at room temperature $\left(28^{\circ} \mathrm{C}\right)$. Reaction was monitored using TLC. On complete disappearance of the propargyl carbonate, stirring was stopped and the solvent was removed under vacuum. The residue was extracted with a 9:1 mixture of dichloromethane and diethyl ether three times and filtered over a Celite pad. The compounds were separated by flash column chromatography on silica gel (230-400 mesh) using ethylacetate/hexane solvent system.

\section{General procedure for the preparation of $7 a-h$}

To a well-stirred solution of the amino alcohol, or amino phenol (5 mmol) and triethylamine (11 mmol $)$ in anhydrous dichloromethane $(20 \mathrm{~mL})$ at $-78{ }^{\circ} \mathrm{C}$, $\mathrm{PocCl}, \mathbf{1}(10.5 \mathrm{mmol})$ was added dropwise. The reaction mixture was stirred at the same temperature till the completion of the reaction (as shown by TLC). It was then washed with water and dried over anhydrous $\mathrm{Na}_{2} \mathrm{SO}_{4}$. The deprotected compounds $\mathbf{7 a - h}$ were then purified using flash column chromatography on a silica gel (230-400 mesh) column using ethylacetate/hexane solution of appropriate concentration.

Compound 7a:<smiles>C#CCOC(=O)NC(CC)COC(=O)OCC#C</smiles>

Pale yellow oil. ${ }^{1} \mathrm{H}$ NMR $\left(\mathrm{CDCl}_{3}\right): \delta 5.2(\mathrm{db}, \mathrm{J}=8.4,1 \mathrm{H}), 4.7(\mathrm{~d}, \mathrm{~J}=2.4,2 \mathrm{H}), 4.7(\mathrm{sb}, 2 \mathrm{H}), 4.2(\mathrm{~d}$, $\mathrm{J}=4.2,2 \mathrm{H}), 3.77-3.87(\mathrm{~m}, 1 \mathrm{H}), 2.6(\mathrm{t}, \mathrm{J}=2.4,1 \mathrm{H}), 2.5(\mathrm{t}, \mathrm{J}=2.1,1 \mathrm{H}), 1.48-1.69(\mathrm{~m}, 2 \mathrm{H}), 0.97(\mathrm{t}$, $\mathrm{J}=7.2,3 \mathrm{H}) .{ }^{13} \mathrm{C} \mathrm{NMR}\left(\mathrm{CDCl}_{3}\right): \delta 155.0,154.3,78.0,76.7,75.8,74.6,69.2,55.3,52.4,51.5,24.2$, 
10.1. IR (neat): 3295 (s), 2129 (w), 1747 (s), 1714 (s). ESMS: HRMS calculated for $\mathrm{C}_{12} \mathrm{H}_{15} \mathrm{NO}_{5}+\mathrm{Na}: 276.0848$, found: 276.0851.

Compound 7b:<smiles>C#CCOC(=O)NC(COC(=O)OCC#C)CC(C)C</smiles>

Pale yellow oil. ${ }^{1} \mathrm{H}$ NMR $\left(\mathrm{CDCl}_{3}\right): \delta 4.8(\mathrm{~d}, \mathrm{~J}=7.5,1 \mathrm{H}), 4.7(\mathrm{~d}, \mathrm{~J}=2.7,2 \mathrm{H}), 4.7(\mathrm{~d}, \mathrm{~J}=2.4,2 \mathrm{H})$, 4.12-4.25 (m, 2H), 3.94-4.04 (m, 1H), $2.5(\mathrm{t}, \mathrm{J}=2.7,1 \mathrm{H}), 2.4(\mathrm{t}, \mathrm{J}=2.4,1 \mathrm{H}), 1.62-1.71(\mathrm{~m}, 1 \mathrm{H})$, 1.25-1.50 (m, 2H), $0.94(\mathrm{~d}, \mathrm{~J}=6.6,3 \mathrm{H}), 0.93(\mathrm{~d}, \mathrm{~J}=6.6,3 \mathrm{H}) .{ }^{13} \mathrm{C} \mathrm{NMR}\left(\mathrm{CDCl}_{3}\right): \delta 154.8,154.3$, 78.0, 76.7, 75.8, 74.7, 70.1, 55.4, 52.5, 48.3, 40.1, 24.5, 22.8, 21.8. IR (neat): 3297 (s), 2958 (s), 2129 (w), 1756 (s), 1713 (s). ESMS: HRMS calculated for $\mathrm{C}_{14} \mathrm{H}_{19} \mathrm{NO}_{5}+\mathrm{Na}$ : 304.1161, found: 304.1159.

Compound 7c:<smiles>C#CCOC(=O)NC(COC(=O)OCC#C)Cc1ccccc1</smiles>

Pale yellow oil. ${ }^{1} \mathrm{H}$ NMR $\left(\mathrm{CDCl}_{3}\right): \delta 7.17-7.32(\mathrm{~m}, 5 \mathrm{H}), 5.2(\mathrm{sb}, 1 \mathrm{H}), 4.7(\mathrm{~d}, \mathrm{~J}=2.1,2 \mathrm{H}), 4.6(\mathrm{sb}$, 2H), 4.21-4.09 (m, 3H), 2.81-2.95 (m, 2H), $2.5(\mathrm{t}, \mathrm{J}=2.1,1 \mathrm{H}), 2.5(\mathrm{t}, \mathrm{J}=2.4,1 \mathrm{H}) .{ }^{13} \mathrm{C} \mathrm{NMR}$ $\left(\mathrm{CDCl}_{3}\right): \delta 154.7,154.2,136.5,129.1,128.6,126.8,77.9,76.7,75.9,74.7,68.3,55.5,52.5,51.3$ 37.2. IR (neat): 3293 (m), 3028 (w), 2129 (w), 1753 (s), 1715 (s). ESMS: HRMS calculated for $\mathrm{C}_{17} \mathrm{H}_{17} \mathrm{NO}_{5}+\mathrm{Na}: 338.1004$, found: 338.1002 .

Compound 7d:<smiles>C#CCOC(=O)NC(Cc1ccc(OC(=O)OCC#C)cc1)C(=O)OC</smiles> 
Colorless gummy solid. ${ }^{1} \mathrm{H}$ NMR $\left(\mathrm{CDCl}_{3}\right): \delta 7.1(\mathrm{sb}, 4 \mathrm{H}), 5.4(\mathrm{~d}, \mathrm{~J}=7.8,1 \mathrm{H}), 4.8(\mathrm{~d}, \mathrm{~J}=2.4,2 \mathrm{H})$, 4.60-4.66 (two sets of peaks overlapping, 3H), $3.7(\mathrm{~s}, 3 \mathrm{H}), 3.04-3.18(\mathrm{~m}, 2 \mathrm{H}), 2.6(\mathrm{t}, \mathrm{J}=2.4,1 \mathrm{H})$, $2.5(\mathrm{t}, \mathrm{J}=2.1,1 \mathrm{H}) .{ }^{13} \mathrm{C} \mathrm{NMR}\left(\mathrm{CDCl}_{3}\right): \delta 171.5,154.7,152.9,150.1,133.7,130.3,121.0,77.8$, 76.4, 76.2, 74.9, 55.9, 54.7, 52.8, 52.4, 37.4. IR (neat): 3312 (s), 3031 (w), 2127 (w), 1750 (s), 1710 (s). ESMS: HRMS calculated for $\mathrm{C}_{18} \mathrm{H}_{17} \mathrm{NO}_{7}+\mathrm{Na}$ : 382.0898 , found: 382.0895 .

\section{Compound 7e:}

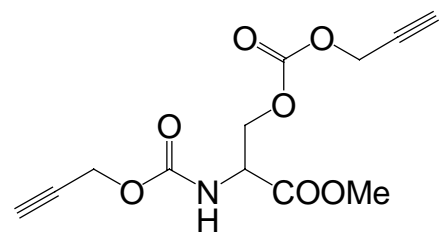

White solid, which decomposes above $200{ }^{\circ} \mathrm{C} .{ }^{1} \mathrm{H}$ NMR $\left(\mathrm{CDCl}_{3}\right): \delta 5.8(\mathrm{~d}, \mathrm{~J}=7.8,1 \mathrm{H}), 4.7(\mathrm{~d}$, $\mathrm{J}=2.4,2 \mathrm{H}), 4.7(\mathrm{~d}, \mathrm{~J}=2.4,2 \mathrm{H}), 4.62-4.67(\mathrm{~m}, 1 \mathrm{H}), 4.45-4.58(\mathrm{~m}, 2 \mathrm{H}), 3.8(\mathrm{~s}, 3 \mathrm{H}), 2.57$ (t, J=2.4, 1H), $2.51(\mathrm{t}, \mathrm{J}=2.4,1 \mathrm{H}) .{ }^{13} \mathrm{C} \mathrm{NMR}\left(\mathrm{CDCl}_{3}\right): \delta 169.1,154.8,153.9,77.7,76.5,76.0,74.9,67.5$, 55.6, 53.2, 53.0, 52.9. IR (KBr): 3304 (s), 2129 (w), 1754 (s), 1714 (s). ESMS: HRMS calculated for $\mathrm{C}_{12} \mathrm{H}_{13} \mathrm{NO}_{7}+\mathrm{Na}: 306.0590$, found: 306.0583 .

\section{Compound 7f:}

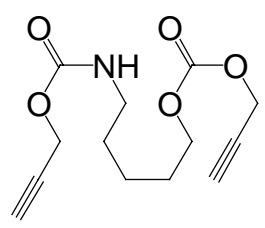

Pale yellow oil. ${ }^{1} \mathrm{H}$ NMR $\left(\mathrm{CDCl}_{3}\right): \delta 5.1(\mathrm{sb}, 1 \mathrm{H}), 4.7(\mathrm{~d}, \mathrm{~J}=2.4,2 \mathrm{H}), 4.6(\mathrm{~d}, \mathrm{~J}=2.1,2 \mathrm{H}), 4.2(\mathrm{t}$, $\mathrm{J}=6.6,2 \mathrm{H}), 3.2(\mathrm{q}, \mathrm{J}=6.6,2 \mathrm{H}), 2.57(\mathrm{t}, \mathrm{J}=2.4,1 \mathrm{H}), 2.50(\mathrm{t}, \mathrm{J}=2.1,1 \mathrm{H}), 1.7$ (qi, J=6.9, 2H), 1.50$1.60(\mathrm{~m}, 2 \mathrm{H}), 1.36-1.46(\mathrm{~m}, 2 \mathrm{H}) .{ }^{13} \mathrm{C} \mathrm{NMR}\left(\mathrm{CDCl}_{3}\right): \delta 155.4,154.4,78.2,76.9,75.6,74.5,68.2$, 55.0, 52.2, 40.7, 29.2, 28.0, 22.7. IR (neat): 3298 (s), 2128 (w), 1752 (s), 1716 (s). ESMS: HRMS calculated for $\mathrm{C}_{13} \mathrm{H}_{17} \mathrm{NO}_{5}+\mathrm{Na}: 290.1004$, found: 290.1008 .

\section{Compound 7g:}




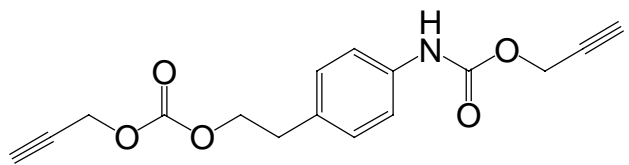

White solid, mp: $107{ }^{\circ} \mathrm{C} .{ }^{1} \mathrm{H}$ NMR $\left(\mathrm{CDCl}_{3}\right): \delta 7.3(\mathrm{~d}, \mathrm{~J}=8.1,2 \mathrm{H}), 7.1(\mathrm{~d}, \mathrm{~J}=8.1,2 \mathrm{H}), 4.7(\mathrm{~d}$, $\mathrm{J}=2.4,2 \mathrm{H}), 4.7(\mathrm{~d}, \mathrm{~J}=2.1,2 \mathrm{H}), 4.3(\mathrm{t}, \mathrm{J}=6.9,2 \mathrm{H}), 2.9(\mathrm{t}, \mathrm{J}=6.9,2 \mathrm{H}), 2.50-2.53$ (two triplets, $2 \mathrm{H})$.

${ }^{13} \mathrm{C} \mathrm{NMR}\left(\mathrm{CDCl}_{3}\right): \delta 154.4,152.5,136.1,132.4,129.5,119.0,77.8,76.9,75.7,75.0,68.8,55.2$, 52.7, 34.3. IR (KBr): 3373 (s), 2132 (w) 1742 (s), 1703 (s). ESMS: HRMS calculated for $\mathrm{C}_{16} \mathrm{H}_{15} \mathrm{NO}_{5}+\mathrm{Na}: 324.0848$, found: 324.0853 .

Compound 7h:<smiles>C#CCOC(=O)Nc1ccc(OC(=O)OCC#C)cc1</smiles>

White solid, mp: $93{ }^{\circ} \mathrm{C} .{ }^{1} \mathrm{H}$ NMR $\left(\mathrm{CDCl}_{3}\right): \delta 7.4(\mathrm{~d}, \mathrm{~J}=8.7,2 \mathrm{H}), 7.1(\mathrm{~d}, \mathrm{~J}=8.7,2 \mathrm{H}), 6.7(\mathrm{sb}, 1 \mathrm{H})$, $4.8(\mathrm{~d}, \mathrm{~J}=2.4,2 \mathrm{H}), 4.7(\mathrm{~d}, \mathrm{~J}=2.4,2 \mathrm{H}), 2.58(\mathrm{t}, \mathrm{J}=2.4,1 \mathrm{H}), 2.523(\mathrm{t}, \mathrm{J}=2.4,1 \mathrm{H}) .{ }^{13} \mathrm{C} \mathrm{NMR}$ $\left(\mathrm{CDCl}_{3}\right): \delta 153.2,152.5,146.7,135.8,121.5,119.7,77.7,76.4,76.2,75.1,56.0,52.8 . \mathrm{IR}(\mathrm{KBr}):$ 3364 (s), 2127 (w), 1748 (s), 1708 (s). ESMS: HRMS calculated for $\mathrm{C}_{14} \mathrm{H}_{11} \mathrm{NO}_{5}+\mathrm{Na}$ : 296.0535, found: 296.0551.

Procedure for the selective deblocking of propargyl carbonates from di-Poc protected amino alcohols and aminophenols

To a stirred solution of $\mathbf{7 a - h}(2 \mathrm{mmol})$ in $\mathrm{CH}_{3} \mathrm{CN}(1.5 \mathrm{~mL})$ at room temperature tetrathiomolybdate 2 (2.2 mmol) was added. The reaction mixture was stirred till the disappearance of the starting material (20-30 min, as shown by TLC). The solvent was removed under vacuum. The residue was extracted with a 9:1 mixture of dichloromethane and diethyl ether three times and filtered over a Celite pad. The compounds, 8a-h were purified by flash column chromatography on silica gel (230-400 mesh) column using hexane-ethyl acetate solvent system.

Compound 8a: 


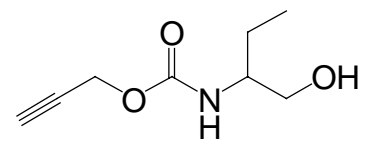

Colorless oil. ${ }^{1} \mathrm{H}$ NMR $\left(\mathrm{CDCl}_{3}\right): \delta 5.3(\mathrm{sb}, 1 \mathrm{H}), 4.6(\mathrm{sb}, 2 \mathrm{H}), 3.55-3.69(\mathrm{~m}, 3 \mathrm{H}), 3.1(\mathrm{sb}, 1 \mathrm{H}), 2.5$ $(\mathrm{sb}, 1 \mathrm{H}), 1.42-1.62(\mathrm{~m}, 2 \mathrm{H}), 0.95(\mathrm{t}, \mathrm{J}=6.9,3 \mathrm{H}) .{ }^{13} \mathrm{C} \mathrm{NMR}\left(\mathrm{CDCl}_{3}\right): \delta 155.8,78.1,74.7,64.5$, 54.7, 52.5, 24.2, 10.3. IR: 3404 (s), 3297 (s), 2966 (m), 2127 (w), 1714 (s), 1697 (s). IR (neat): 3395 (br), 2131(w), 1714 (s). ESMS: HRMS calculated for $\mathrm{C}_{8} \mathrm{H}_{13} \mathrm{NO}_{3}+\mathrm{Na}$ : 194.0793, found: 194.0795.

Compound 8b:<smiles>C#CCOC(=O)NC(CO)CC(C)C</smiles>

Colorless oil. ${ }^{1} \mathrm{H}$ NMR $\left(\mathrm{CDCl}_{3}\right): \delta 5.3(\mathrm{~d}, \mathrm{~J}=8.1,1 \mathrm{H}), 4.6(\mathrm{sb}, 2 \mathrm{H}), 3.64-3.77(\mathrm{~m}, 2 \mathrm{H}), 3.48-3.54$ (m, 1H), $3.2(\mathrm{sb}, 1 \mathrm{H}), 2.5(\mathrm{sb}, 1 \mathrm{H}), 1.59-1.71(\mathrm{~m}, 1 \mathrm{H}), 1.31-1.43(\mathrm{~m}, 2 \mathrm{H}), 0.91-0.93$ (two doublets, $6 \mathrm{H}) .{ }^{13} \mathrm{C} \mathrm{NMR}\left(\mathrm{CDCl}_{3}\right): \delta 155.7,78.1,74.7,65.3,52.5,51.3,40.2,24.6,22.9,22.0 . \mathrm{IR}$ (neat): 3407 (s), 3309 (s), 2128 (w), 1713 (s). ESMS: HRMS calculated for $\mathrm{C}_{10} \mathrm{H}_{17} \mathrm{NO}_{3}+\mathrm{Na}$ : 222.1106, found: 222.1110 .

Compound 8c:

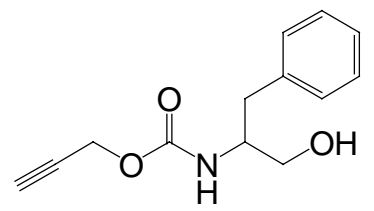

White solid, mp: $66{ }^{\circ} \mathrm{C} .{ }^{1} \mathrm{H}$ NMR $\left(\mathrm{CDCl}_{3}\right): \delta$ 7.18-7.28 (m, 2H), $5.64(\mathrm{sb}, 1 \mathrm{H}), 4.65-4.73(\mathrm{~m}$, $1 \mathrm{H}), 4.6(\mathrm{sb}, 2 \mathrm{H}), 3.9(\mathrm{sb}, 1 \mathrm{H}), 3.48-3.63(\mathrm{~m}, 2 \mathrm{H}), 2.8(\mathrm{~d}, \mathrm{~J}=6.9,2 \mathrm{H}), 2.5(\mathrm{t}, \mathrm{J}=2.4,1 \mathrm{H}) .{ }^{13} \mathrm{C}$ $\operatorname{NMR}\left(\mathrm{CDCl}_{3}\right): \delta 155.4,137.5,129.1,128.3,126.3,78.0,74.7,63.0,54.0,52.3,37.0 . \mathrm{IR}(\mathrm{KBr})$ 3399 (s), 3295 (s), 2129 (w), 1711 (s). ESMS: HRMS calculated for $\mathrm{C}_{13} \mathrm{H}_{15} \mathrm{NO}_{3}+\mathrm{Na}$ : 256.0950, found: 256.0948 .

\section{Compound 8d:}




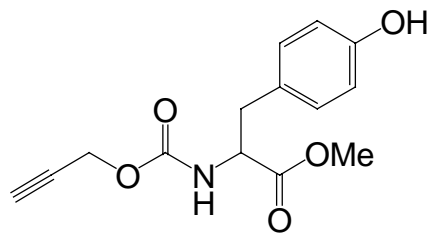

Pale yellow oil. ${ }^{1} \mathrm{H}$ NMR $\left(\mathrm{CDCl}_{3}\right): \delta 6.9(\mathrm{~d}, \mathrm{~J}=8.1,2 \mathrm{H}), 6.7(\mathrm{~d}, \mathrm{~J}=8.1,2 \mathrm{H}), 5.5(\mathrm{~d}, \mathrm{~J}=8.4,1 \mathrm{H}), 4.6$ $(\mathrm{d}, \mathrm{J}=2.4,2 \mathrm{H}), 4.55-4.60(\mathrm{~m}, 1 \mathrm{H}), 3.7(\mathrm{~s}, 3 \mathrm{H}), 2.95-3.08(\mathrm{~m}, 2 \mathrm{H}), 2.5(\mathrm{t}, \mathrm{J}=2.4,1 \mathrm{H}), 2.3(\mathrm{sb}, 1 \mathrm{H})$. ${ }^{13} \mathrm{C} \mathrm{NMR}\left(\mathrm{CDCl}_{3}\right): \delta 172.2,155.2,155.0,130.3,126.9,115.6,77.8,75.0,55.0,52.9,52.5,37.2$. IR (neat): 3347 (br), 3031 (w), 2127 (w), 1710 (s). ESMS: HRMS calculated for $\mathrm{C}_{14} \mathrm{H}_{15} \mathrm{NO}_{5}+\mathrm{Na}$ : 300.0848, found: 300.0847 .

\section{Compound 8e:}<smiles>C#CCOC(=O)NC(CO)C(C)=O</smiles>

Colorless gum. ${ }^{1} \mathrm{H}$ NMR $\left(\mathrm{CDCl}_{3}\right): \delta 6.1(\mathrm{db}, \mathrm{J}=7.5,1 \mathrm{H}), 4.64-4.77(\mathrm{~m}, 2 \mathrm{H}), 4.41-4.46(\mathrm{~m}, 1 \mathrm{H})$, 3.85-4.02 (m, 2H), $3.8(\mathrm{~s}, 3 \mathrm{H}), 3.2(\mathrm{sb}, 1 \mathrm{H}), 2.5(\mathrm{t}, \mathrm{J}=2.4,1 \mathrm{H}) .{ }^{13} \mathrm{C}$ NMR $\left(\mathrm{CDCl}_{3}\right): \delta 170.9$, 155.4, 77.8, 74.9, 62.8, 56.0, 52.84, 52.71. IR (neat): 3414 (s), 3298 (s), 2129 (w), 1713 (s). ESMS: HRMS calculated for $\mathrm{C}_{8} \mathrm{H}_{11} \mathrm{NO}_{5}+\mathrm{Na}$ : 224.0535, found: 224.0536.

\section{Compound 8f:}

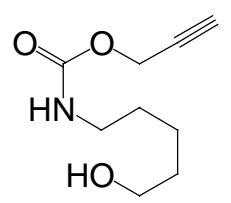

Gummy solid. ${ }^{1} \mathrm{H}$ NMR $\left(\mathrm{CDCl}_{3}\right): \delta 5.4(\mathrm{sb}, 1 \mathrm{H}), 4.6(\mathrm{~d}, \mathrm{~J}=2.1,2 \mathrm{H}), 3.6(\mathrm{t}, \mathrm{J}=6.6,2 \mathrm{H}), 3.18(\mathrm{q}$, $\mathrm{J}=6.6,2 \mathrm{H}), 2.9(\mathrm{sb}, 1 \mathrm{H}), 2.5(\mathrm{t}, \mathrm{J}=2.1,1 \mathrm{H}), 1.33-1.61(\mathrm{~m}, 6 \mathrm{H}) .{ }^{13} \mathrm{C} \mathrm{NMR}\left(\mathrm{CDCl}_{3}\right): \delta 155.6,78.2$, 74.5, 62.1, 52.2, 40.8, 31.9, 29.3, 22.7. IR (neat): 3408 (s), 3297 (s), 2128 (w), 1714 (s). ESMS: HRMS calculated for $\mathrm{C}_{9} \mathrm{H}_{15} \mathrm{NO}_{3}+\mathrm{Na}$ : 208.0950, found: 208.0948 .

Compound 8g: 
<smiles>C#CCOC(=O)Nc1ccc(CCO)cc1</smiles>

White solid, mp: $82{ }^{\circ} \mathrm{C} .{ }^{1} \mathrm{H}$ NMR $\left(\mathrm{CDCl}_{3}\right): \delta 7.3(\mathrm{~d}, \mathrm{~J}=8.1,2 \mathrm{H}), 7.2(\mathrm{~d}, \mathrm{~J}=8.4,2 \mathrm{H}), 4.7(\mathrm{~d}, \mathrm{~J}=2.1$, 2H), $3.8(\mathrm{t}, \mathrm{J}=6.6,2 \mathrm{H}), 2.8(\mathrm{t}, \mathrm{J}=6.6,2 \mathrm{H}), 2.5(\mathrm{t}, \mathrm{J}=2.1,1 \mathrm{H}), 1.5(\mathrm{sb}, 1 \mathrm{H}) .{ }^{13} \mathrm{C} \mathrm{NMR}\left(\mathrm{CDCl}_{3}\right): \delta$ 152.7, 135.8, 134.0, 129.5, 119.1, 77.8, 75.0, 63.5, 52.6, 38.4. IR (KBr): 3398 (br), 2132 (w) 1742 (s), 1702 (s). ESMS: HRMS calculated for $\mathrm{C}_{12} \mathrm{H}_{13} \mathrm{NO}_{3}+\mathrm{Na}$ : 242.0793, found: 242.0788.

\section{Compound 8h:}

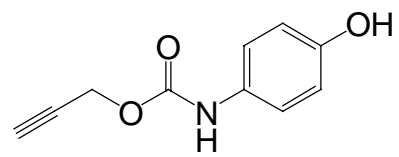

White solid, mp: $132{ }^{\circ} \mathrm{C} .{ }^{1} \mathrm{H}$ NMR $\left(\mathrm{CDCl}_{3}\right): \delta 8.6(\mathrm{sb}, 1 \mathrm{H}), 7.2(\mathrm{~d}, \mathrm{~J}=8.4,2 \mathrm{H}), 6.7(\mathrm{~d}, \mathrm{~J}=8.4,2 \mathrm{H})$, $4.7(\mathrm{~d}, \mathrm{~J}=2.4,2 \mathrm{H}), 2.8(\mathrm{sb}, 1 \mathrm{H}), 2.5(\mathrm{t}, \mathrm{J}=2.4,1 \mathrm{H}) .{ }^{13} \mathrm{C} \mathrm{NMR}\left(\mathrm{CDCl}_{3}\right): \delta 152.6,129.6,120.1$, 114.9, 77.9, 74.4, 51.4. IR (KBr): 3378 (br), 2127 (w), 1705 (s). ESMS: HRMS calculated for $\mathrm{C}_{10} \mathrm{H}_{9} \mathrm{NO}_{3}+\mathrm{Na}: 192.0660$, found: 192.0657. 
NMR spectra for compounds $4 a-h$

Compound 4a
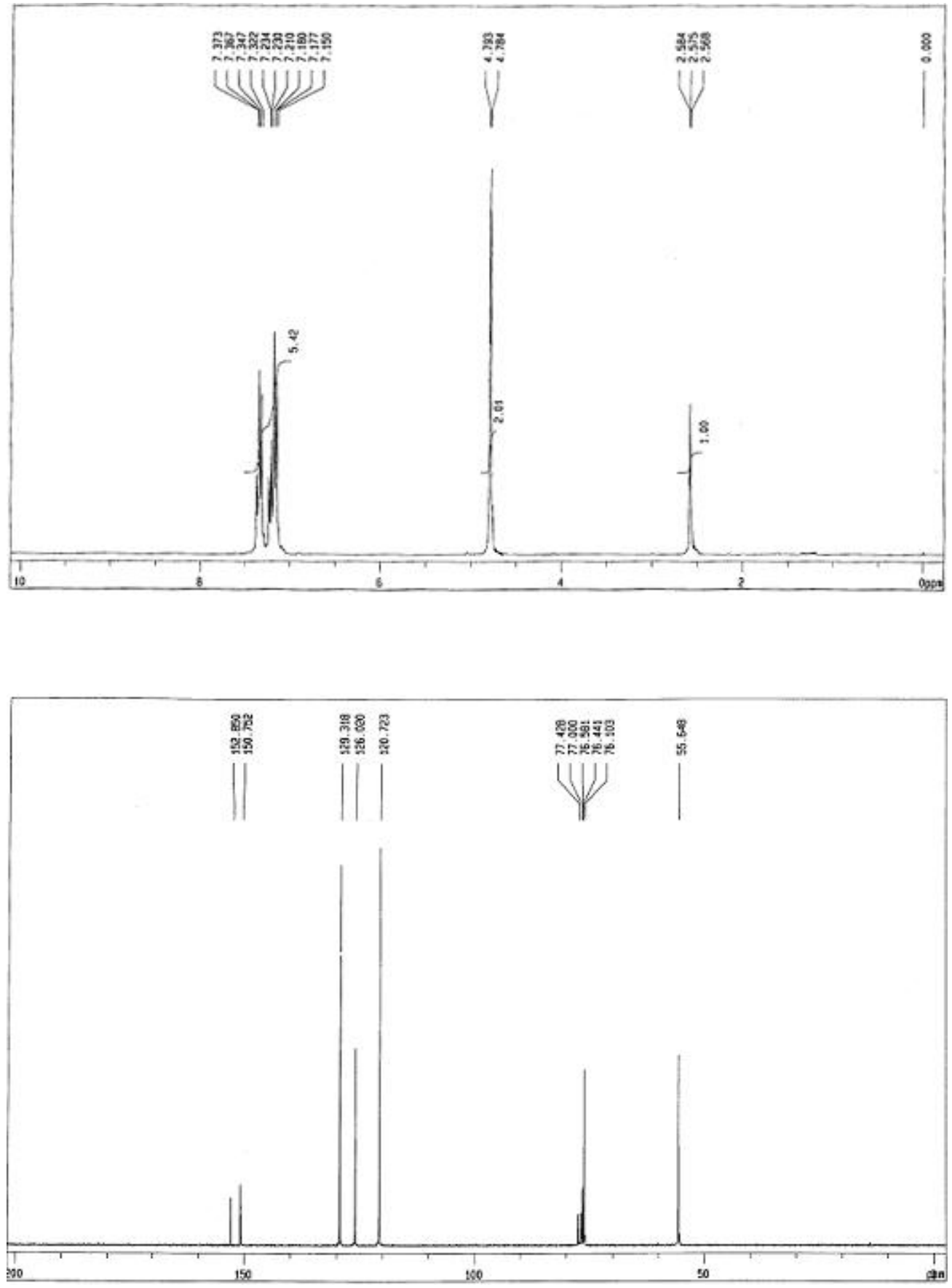
Compound 4b
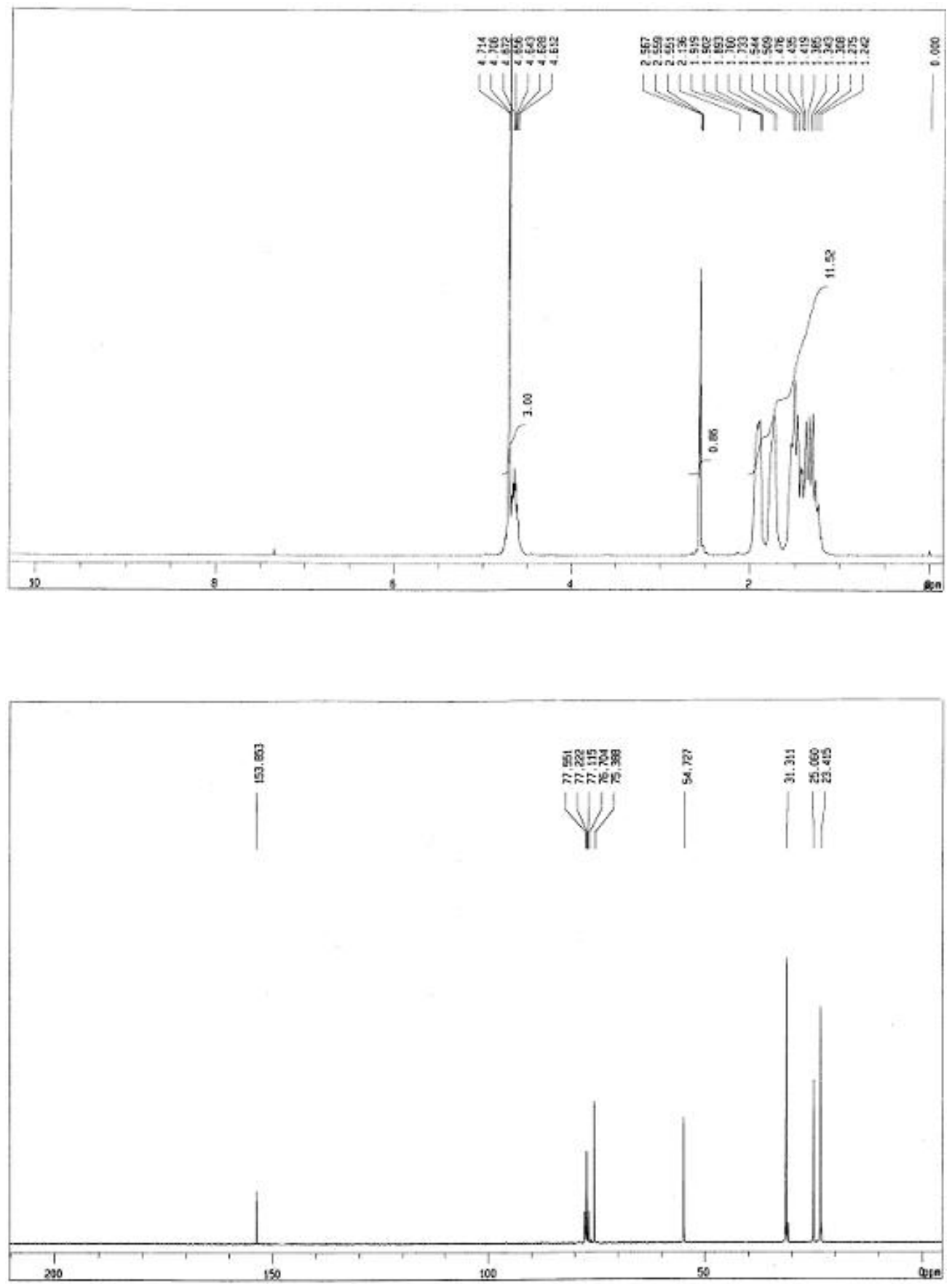
Compound 4c
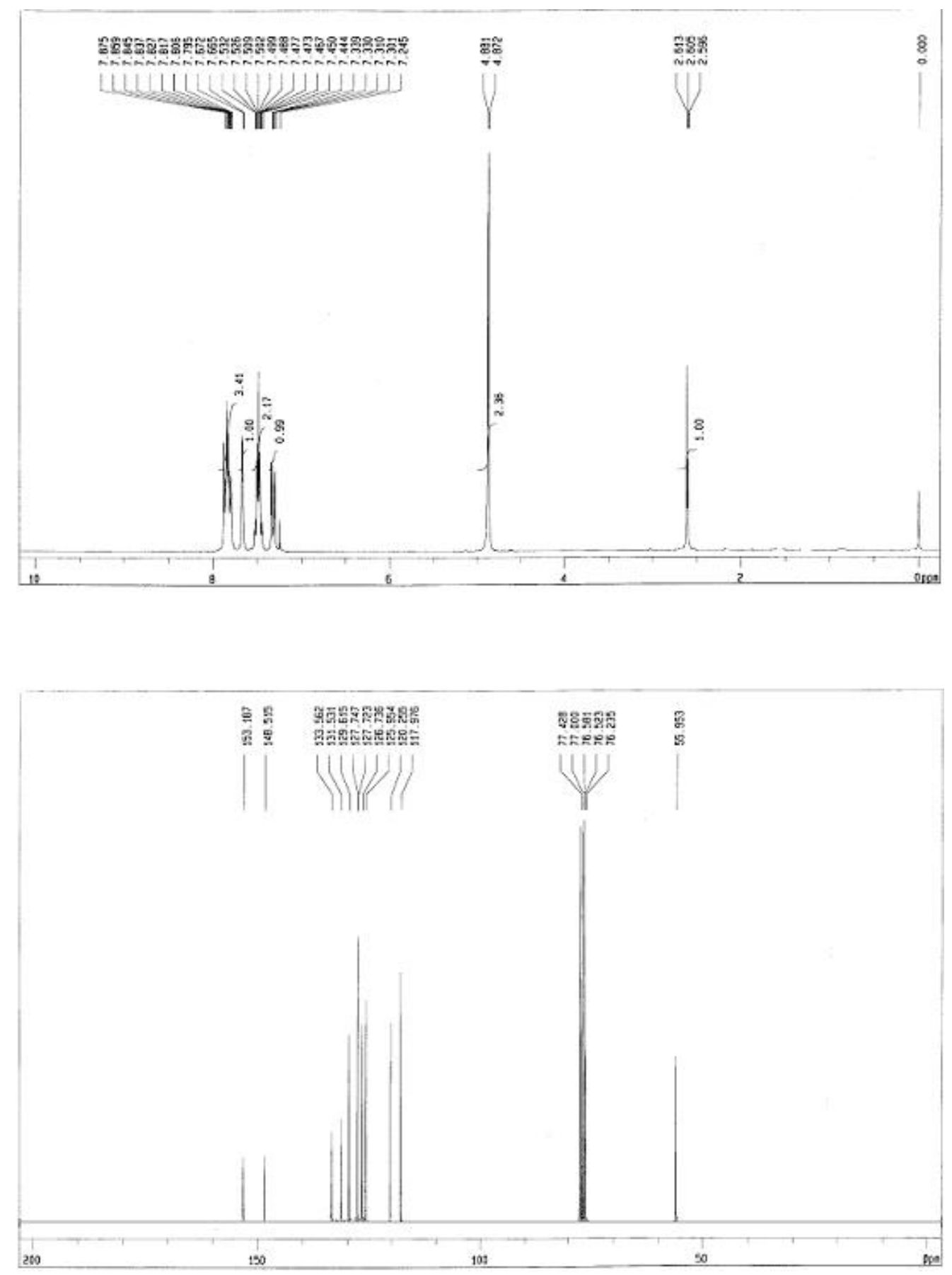

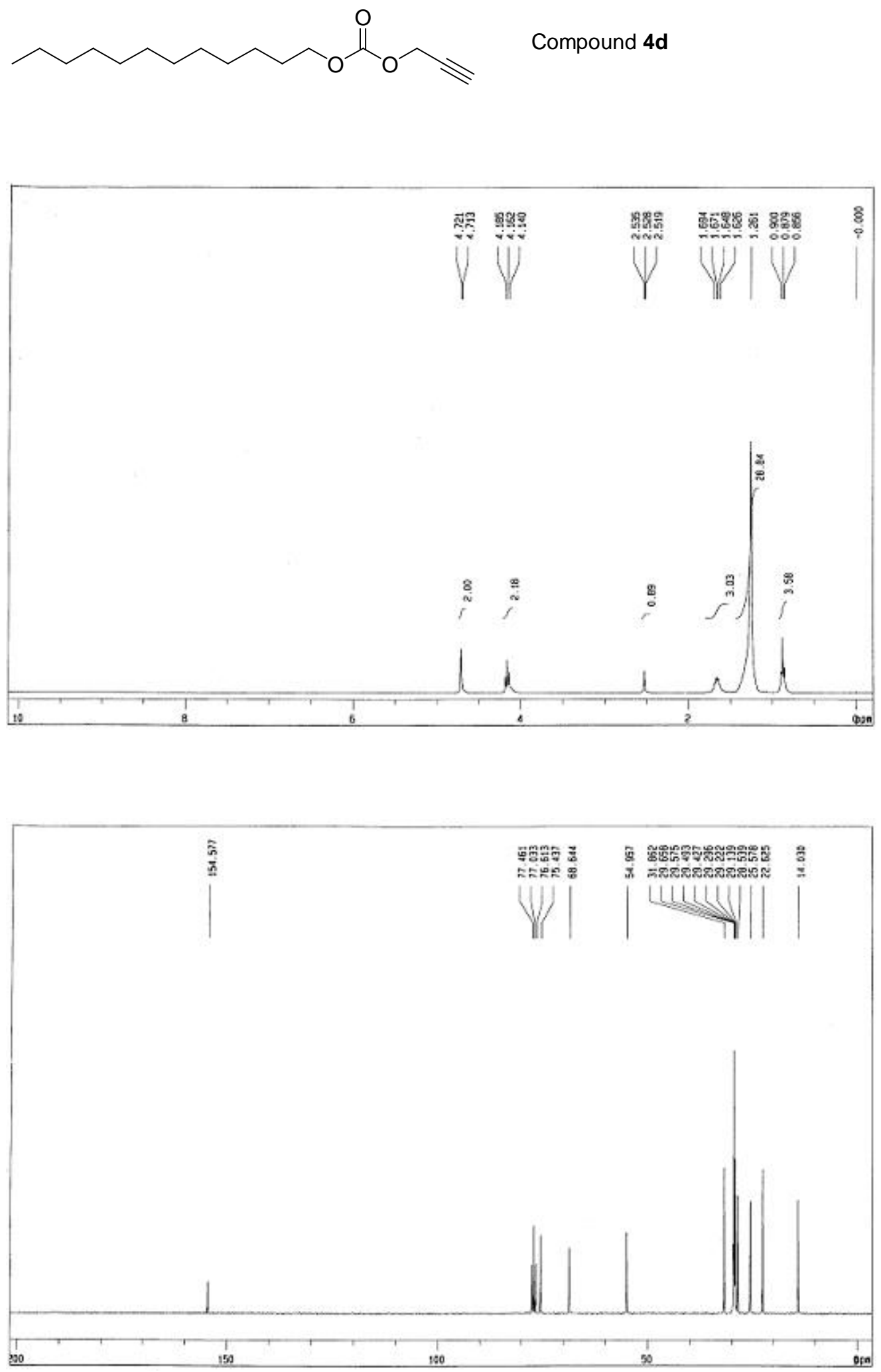
$\overbrace{\mathrm{H}}$
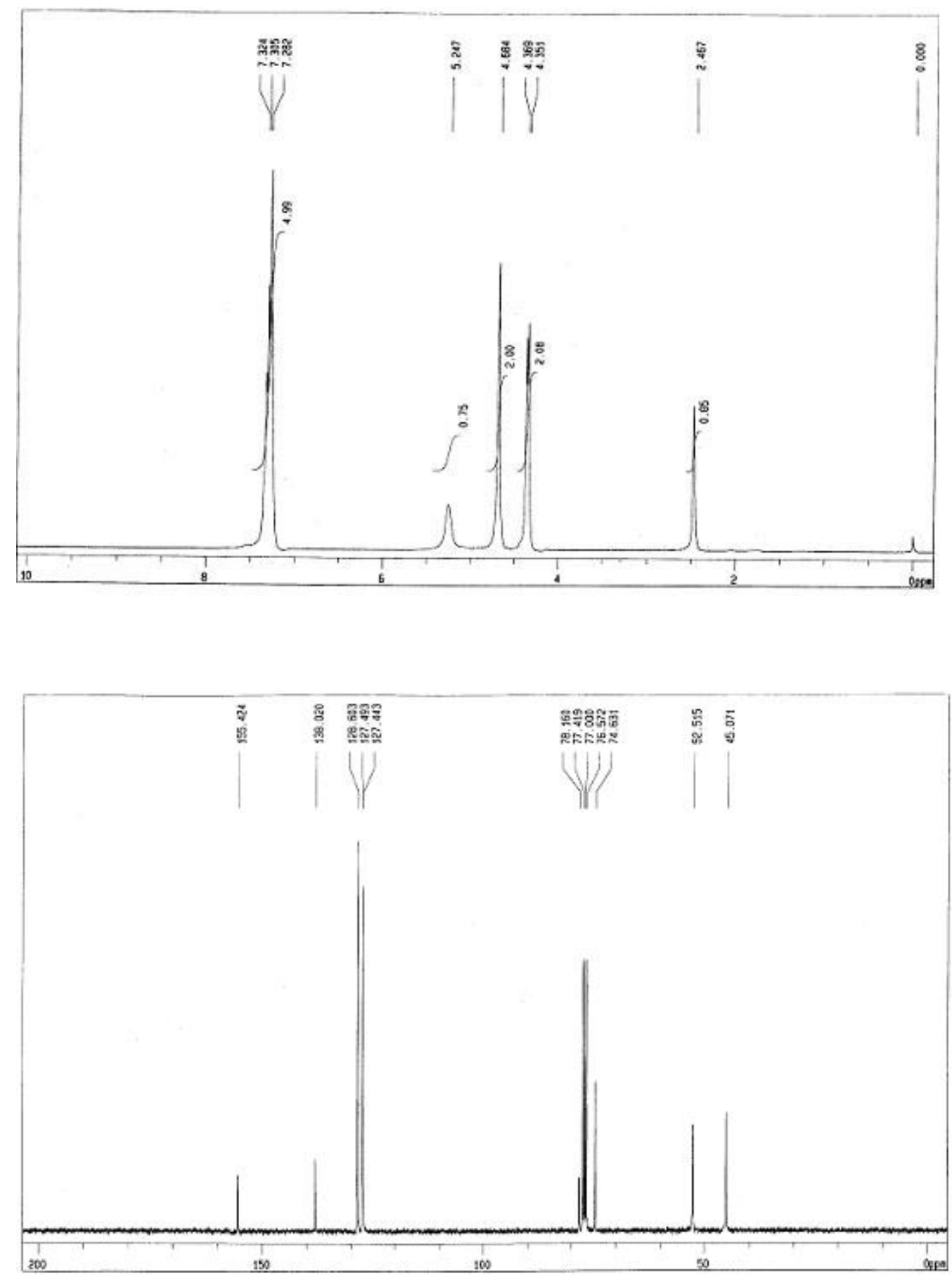
Compound 4f
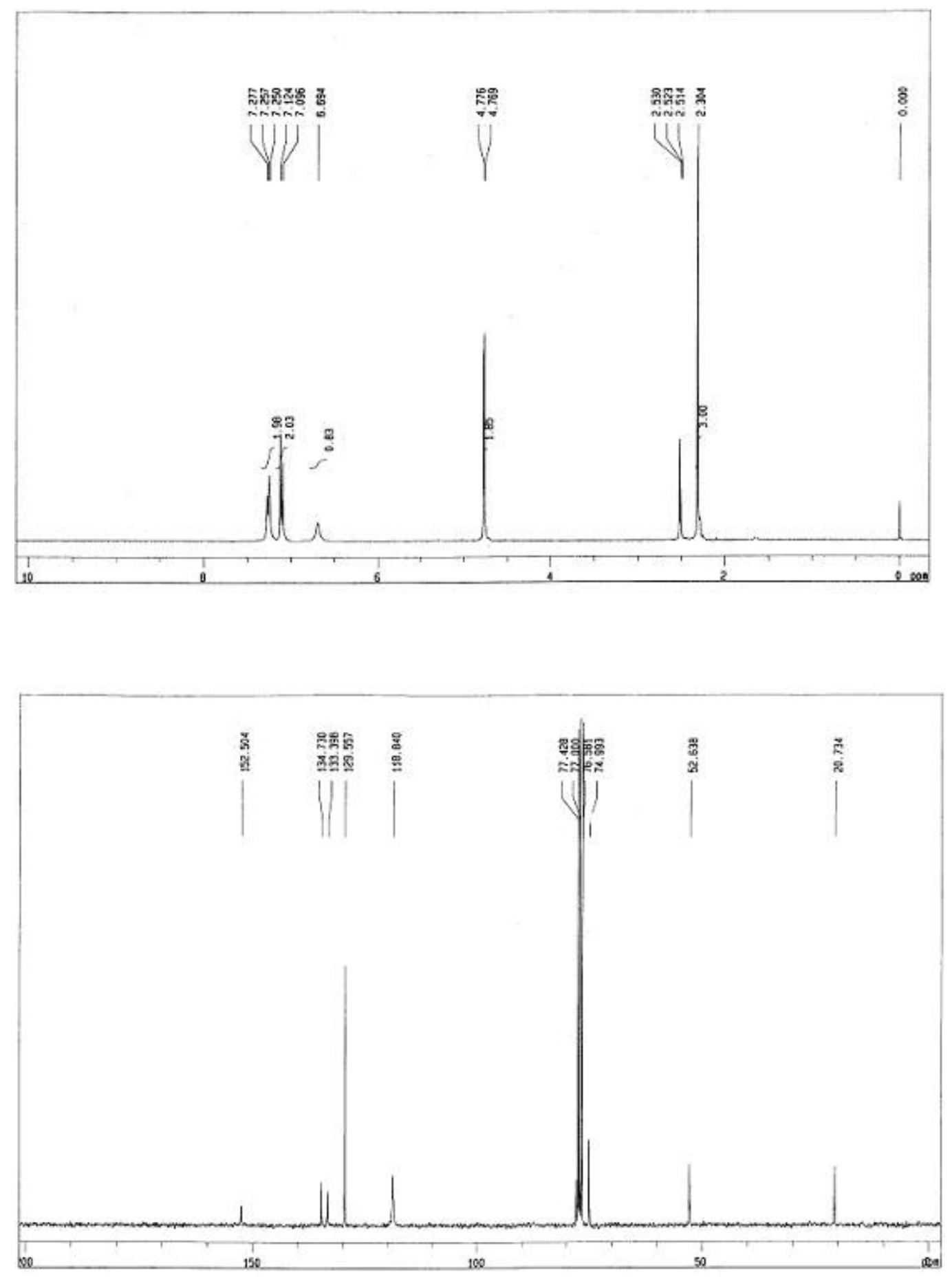
$\sim$ Compound $\mathbf{4 g}$
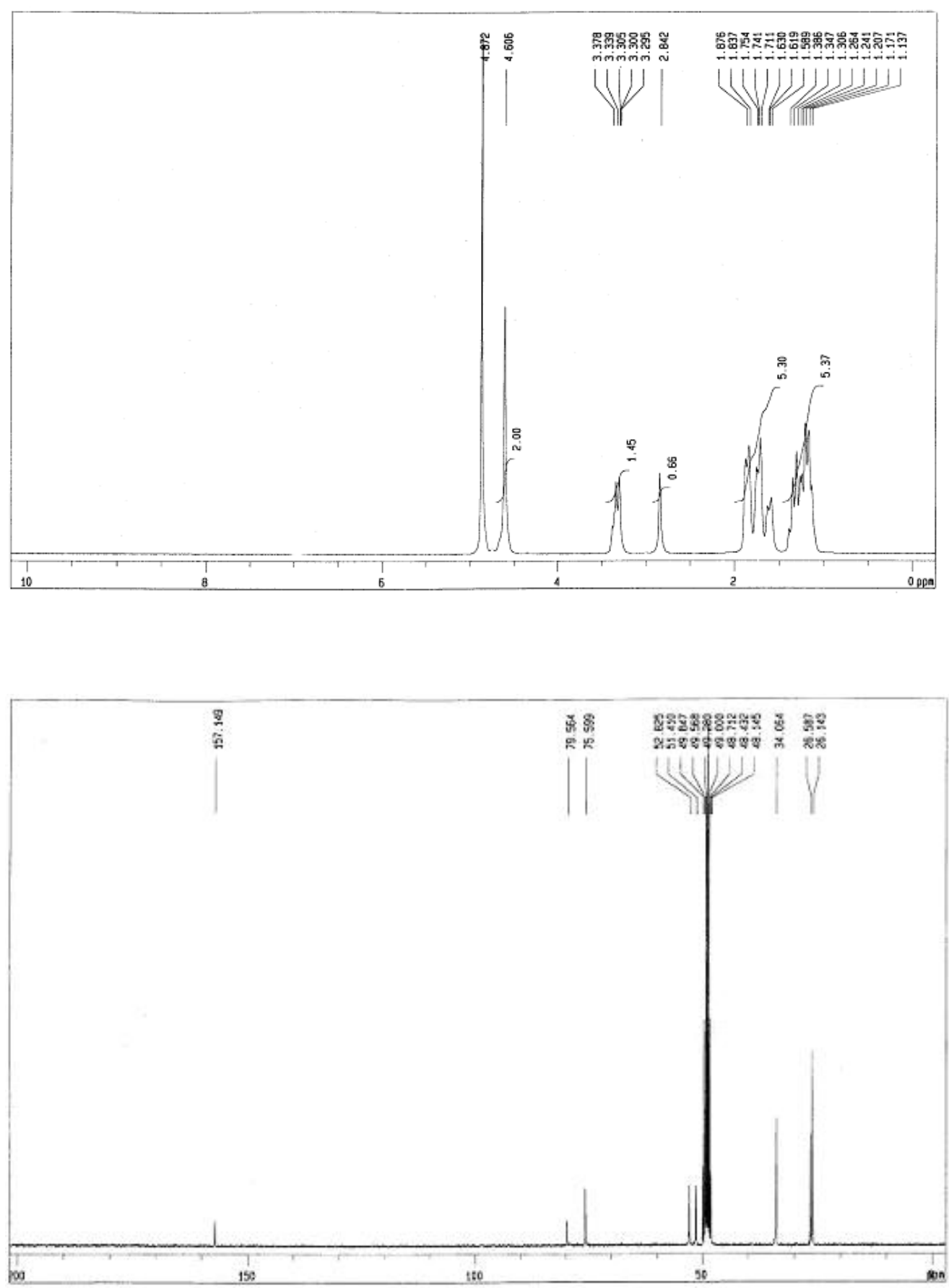
$\overbrace{\text { Compound } 4 h}$
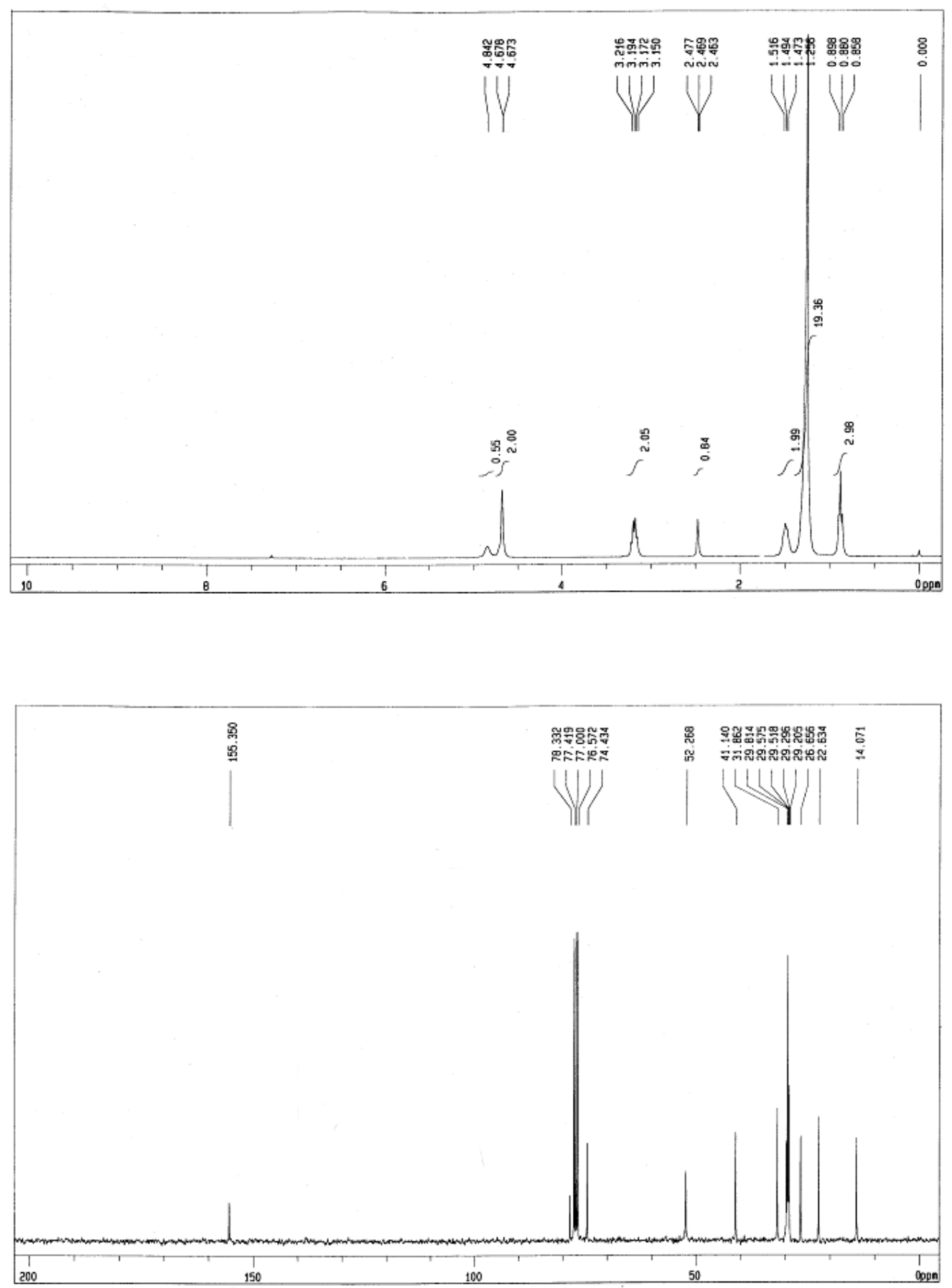
NMR spectra for compounds $7 a-h$<smiles>C#CCOC(=O)NC(CC)COC(=O)OCC#C</smiles>

Compound 7a
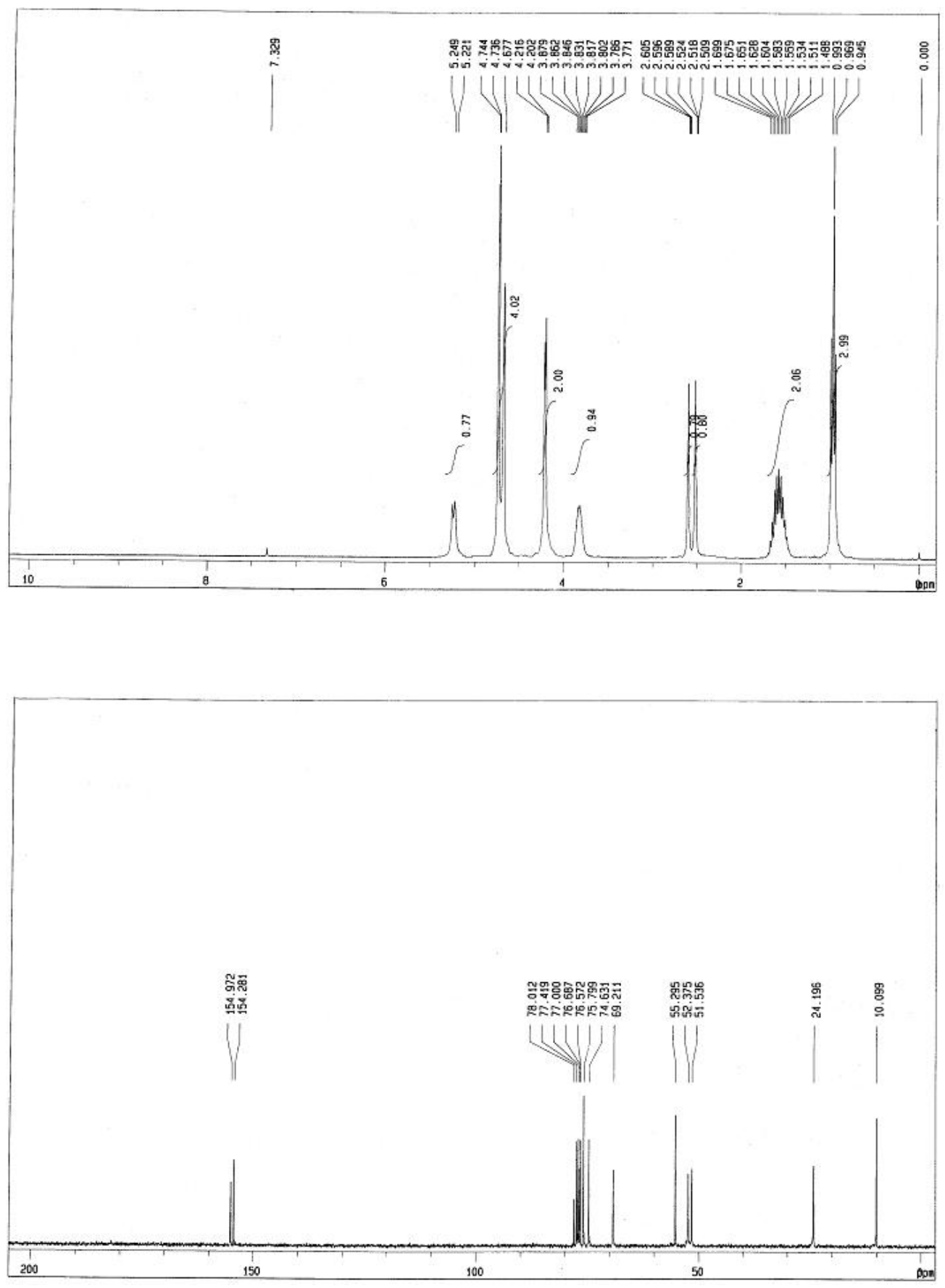
<smiles>C#CCOC(=O)NC(COC(=O)OCC#C)CC(C)C</smiles>

Compound $\mathbf{7 b}$
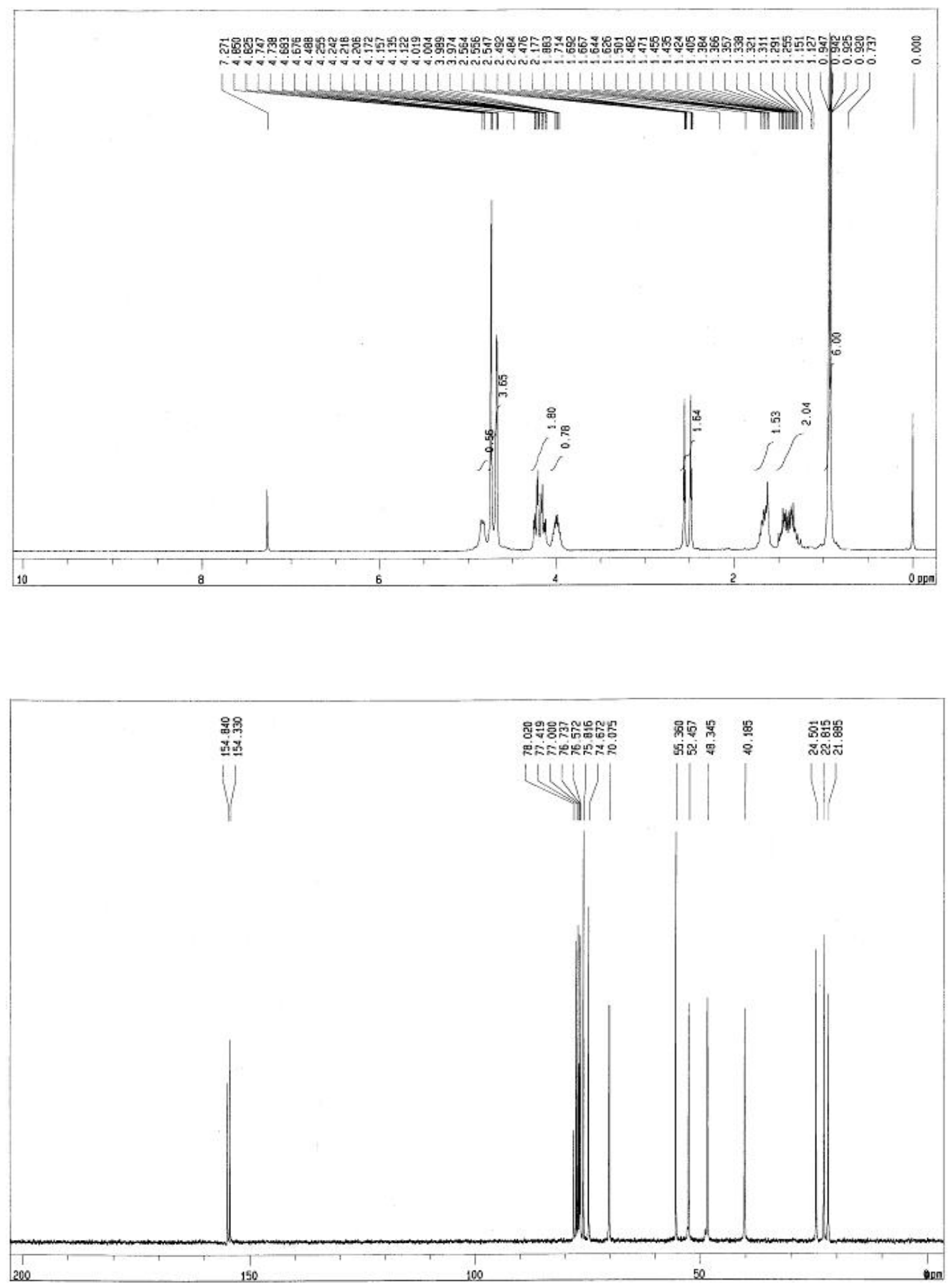
<smiles>C#CCOC(=O)NC(COC(=O)OCC#C)Cc1ccccc1</smiles>
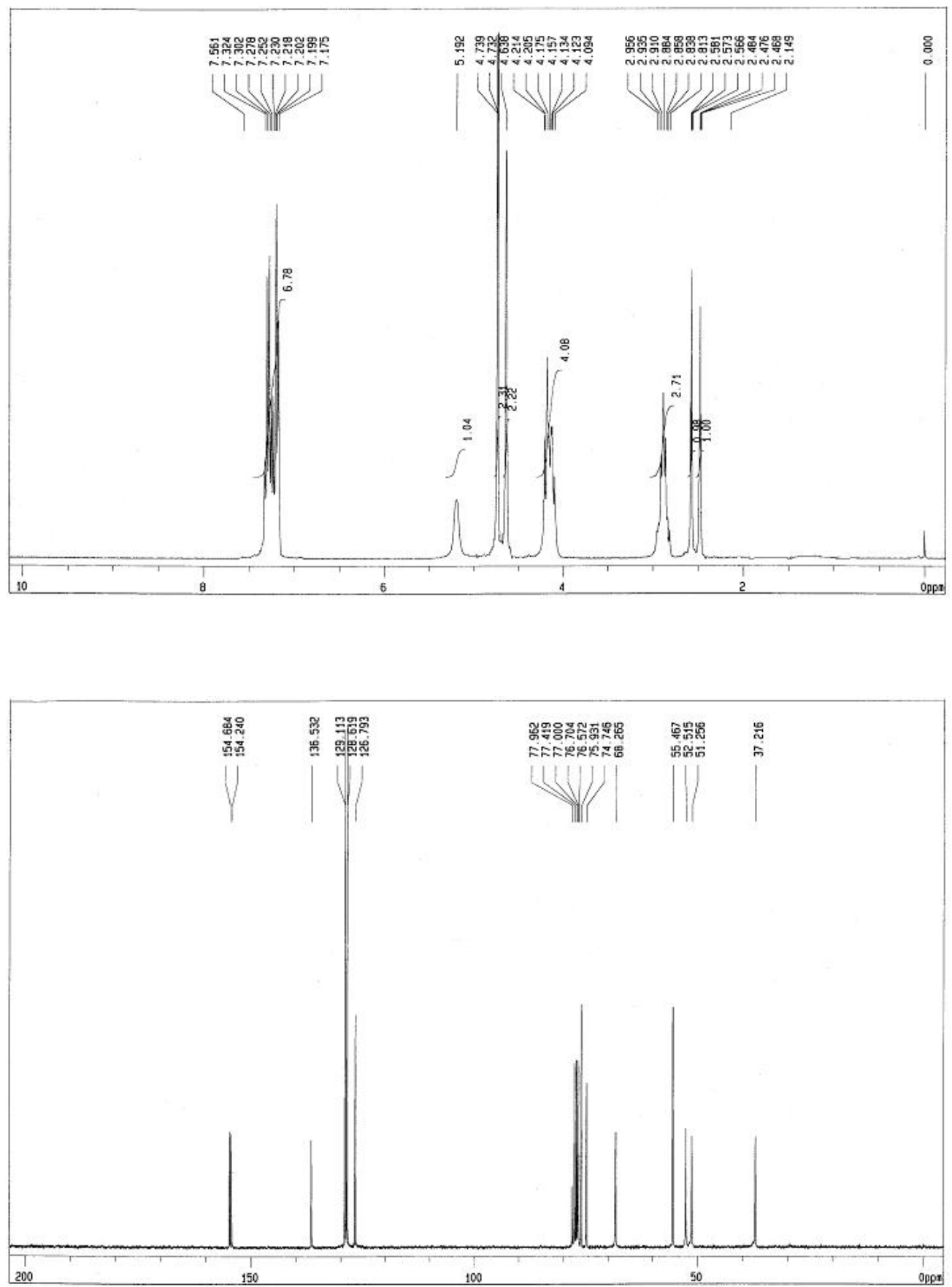
<smiles>C#CCOC(=O)NC(Cc1ccc(OC(=O)OCC#C)cc1)C(=O)OC</smiles>

Compound 7d
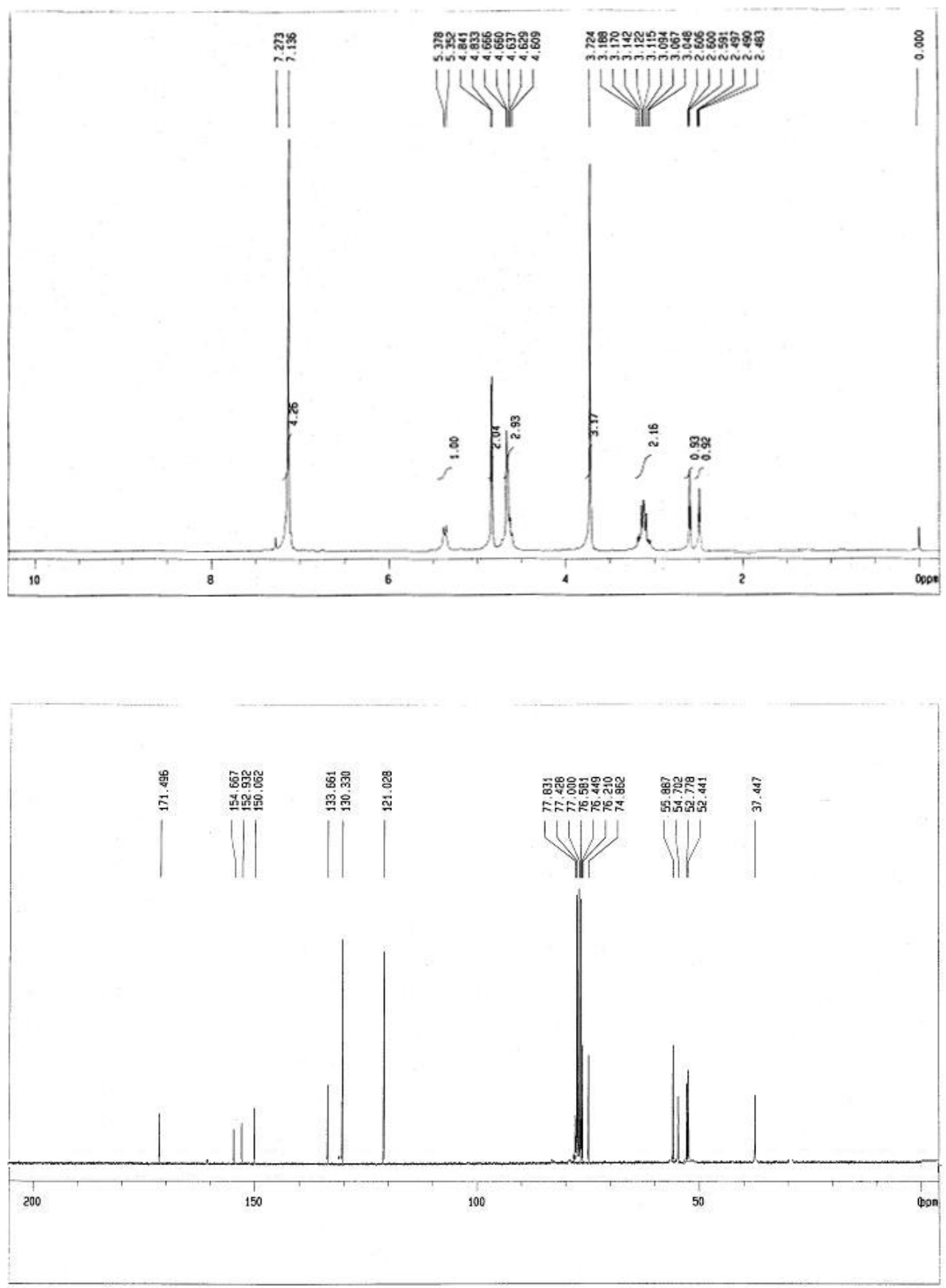
<smiles>C#CCOC(=O)NC(COC(=O)OCC#C)C(=O)OC</smiles>
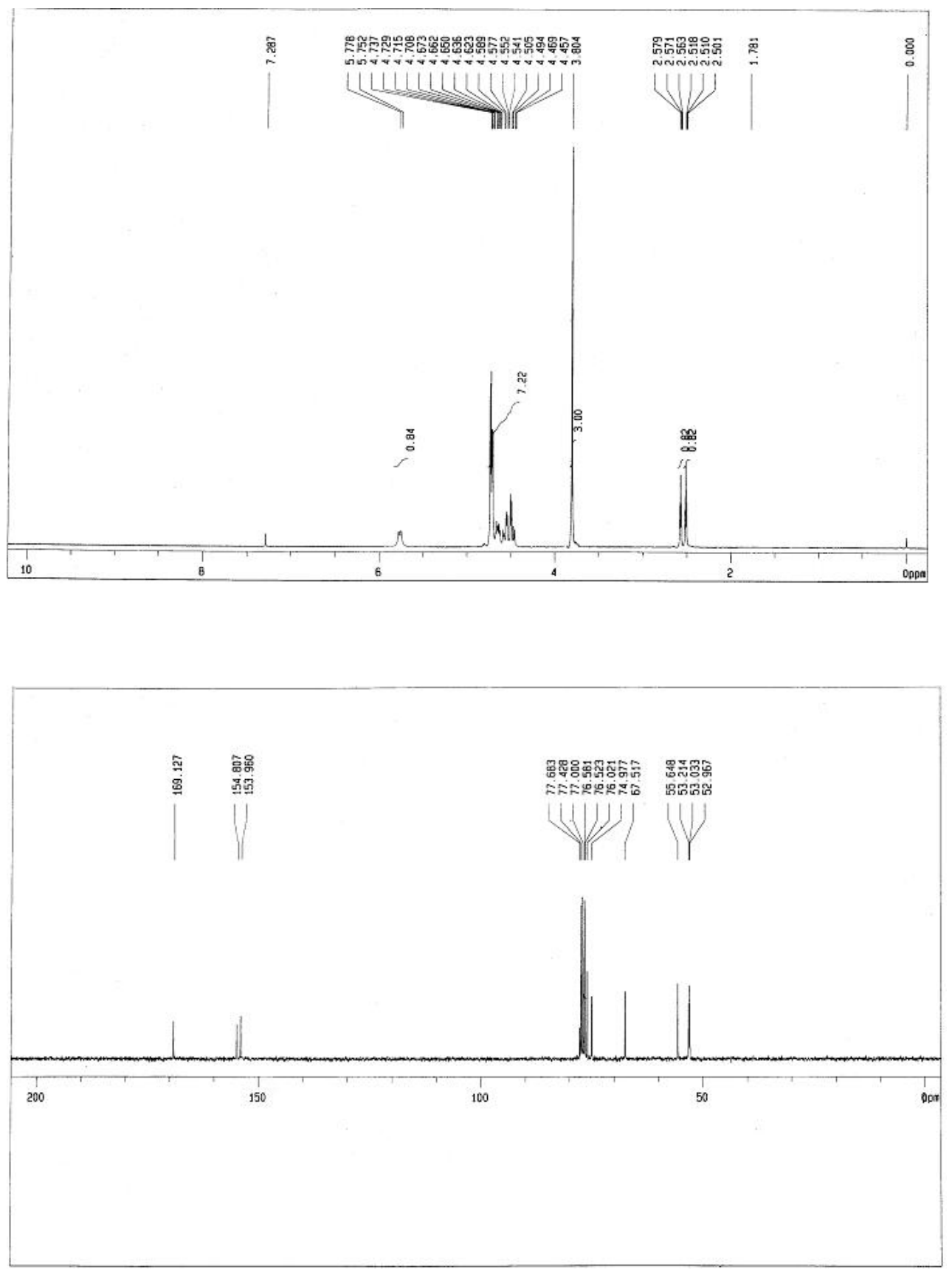
<smiles>C#CCOC(=O)NCCCCCOC(=O)OCC#C</smiles>
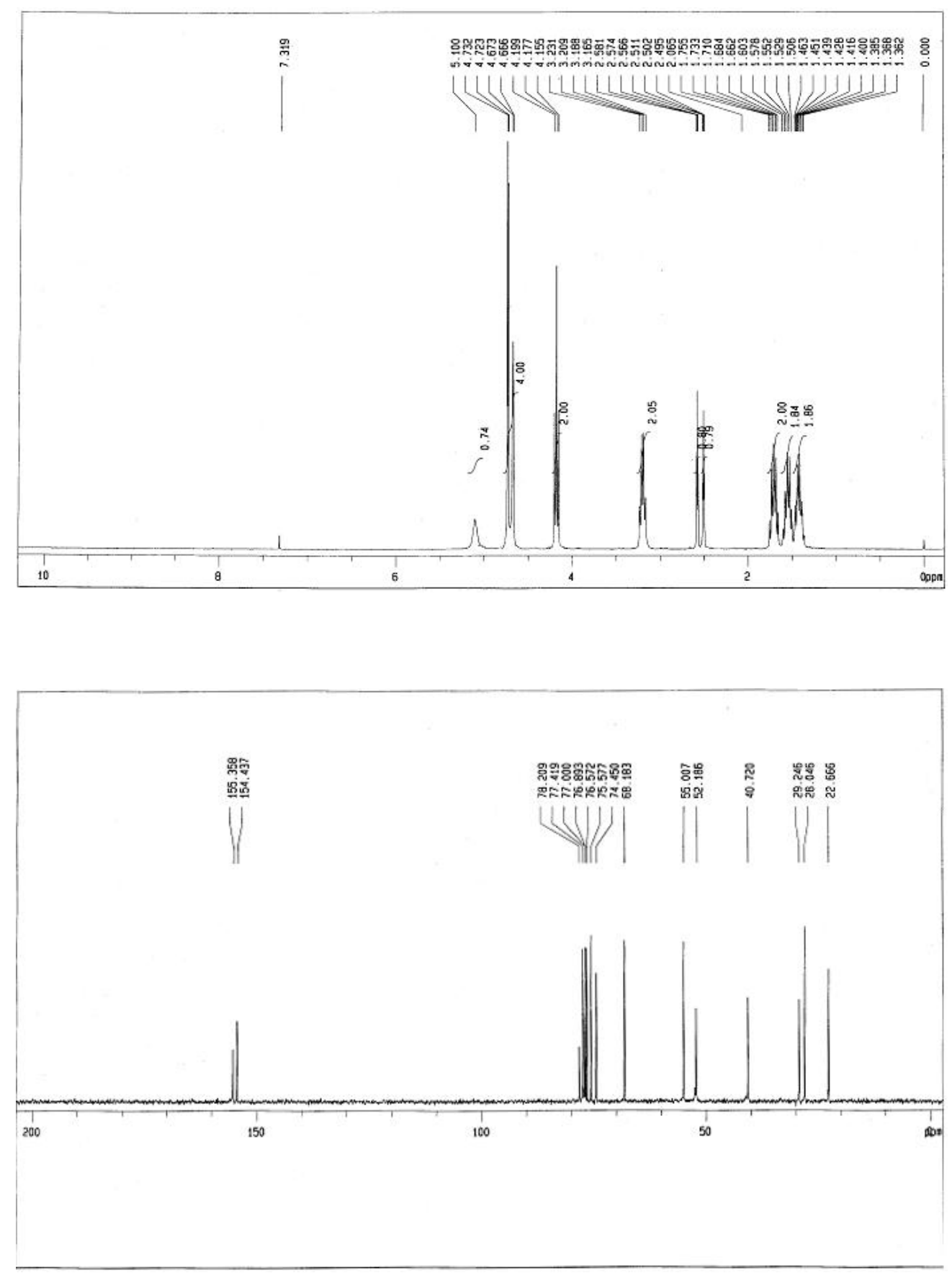
<smiles>C#CCOC(=O)Nc1ccc(CCOC(=O)OCC#C)cc1</smiles>
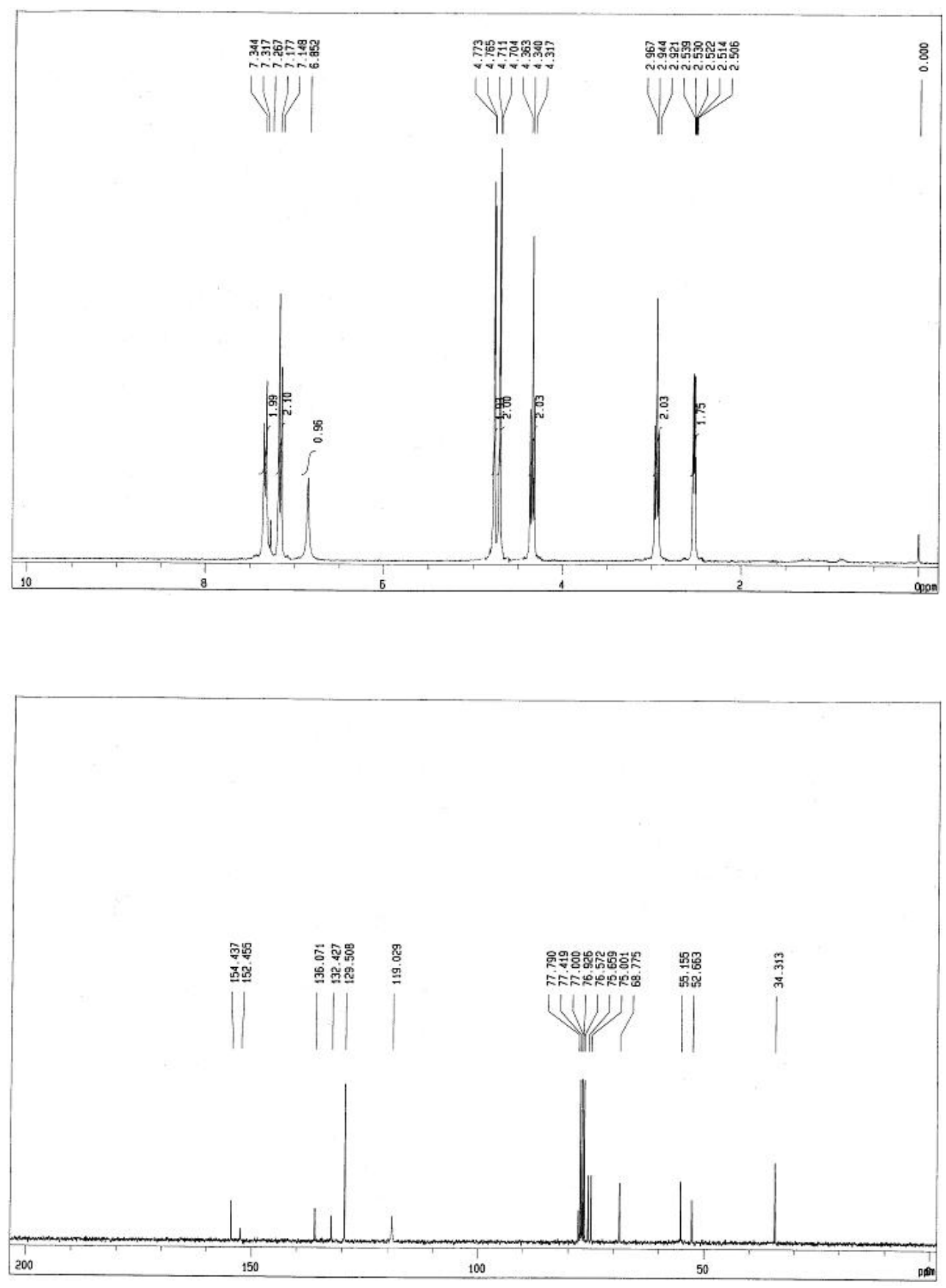
Compound $7 \mathrm{~h}$
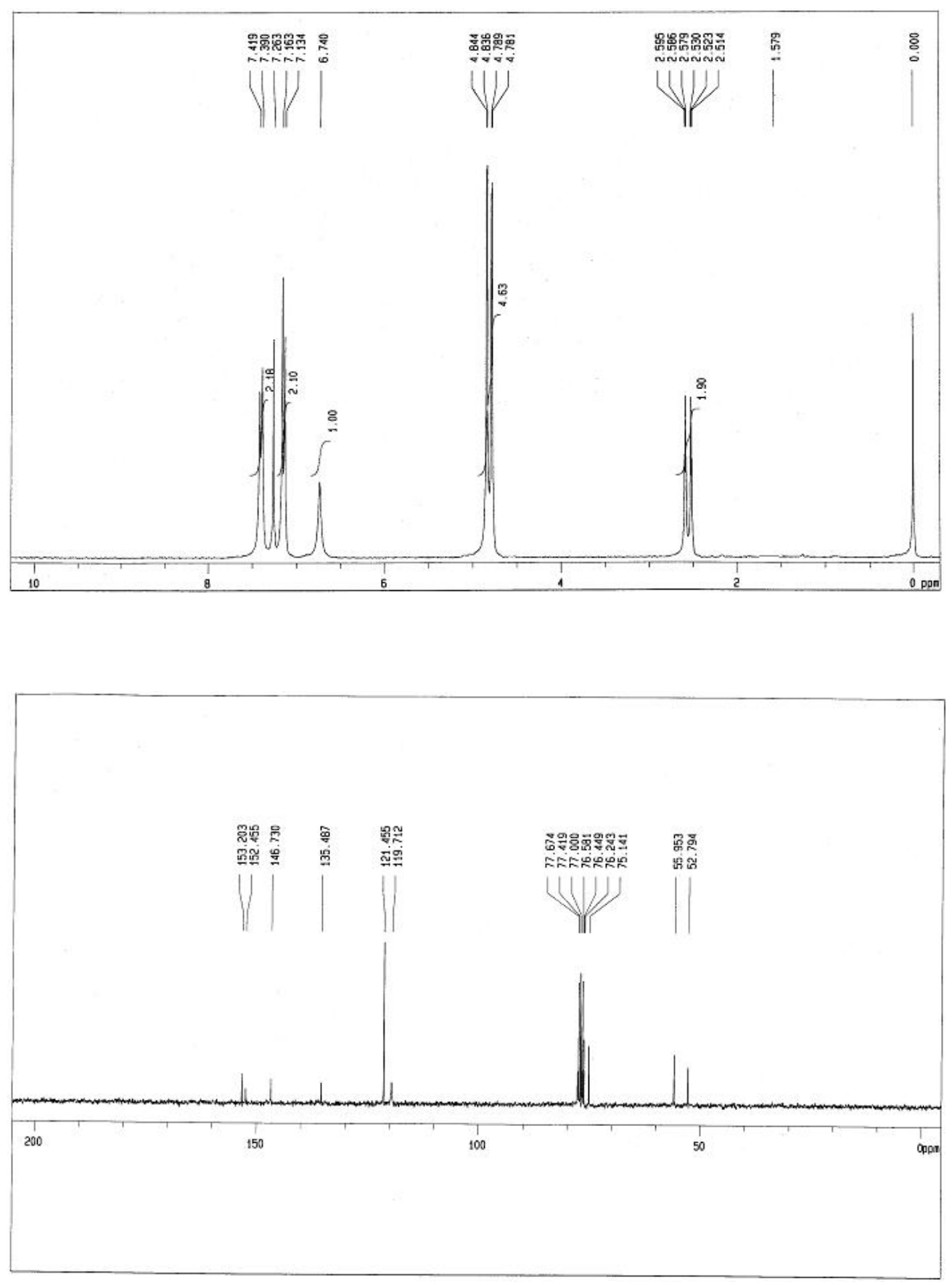
NMR spectra for compounds $8 a-h$

Compound 8a
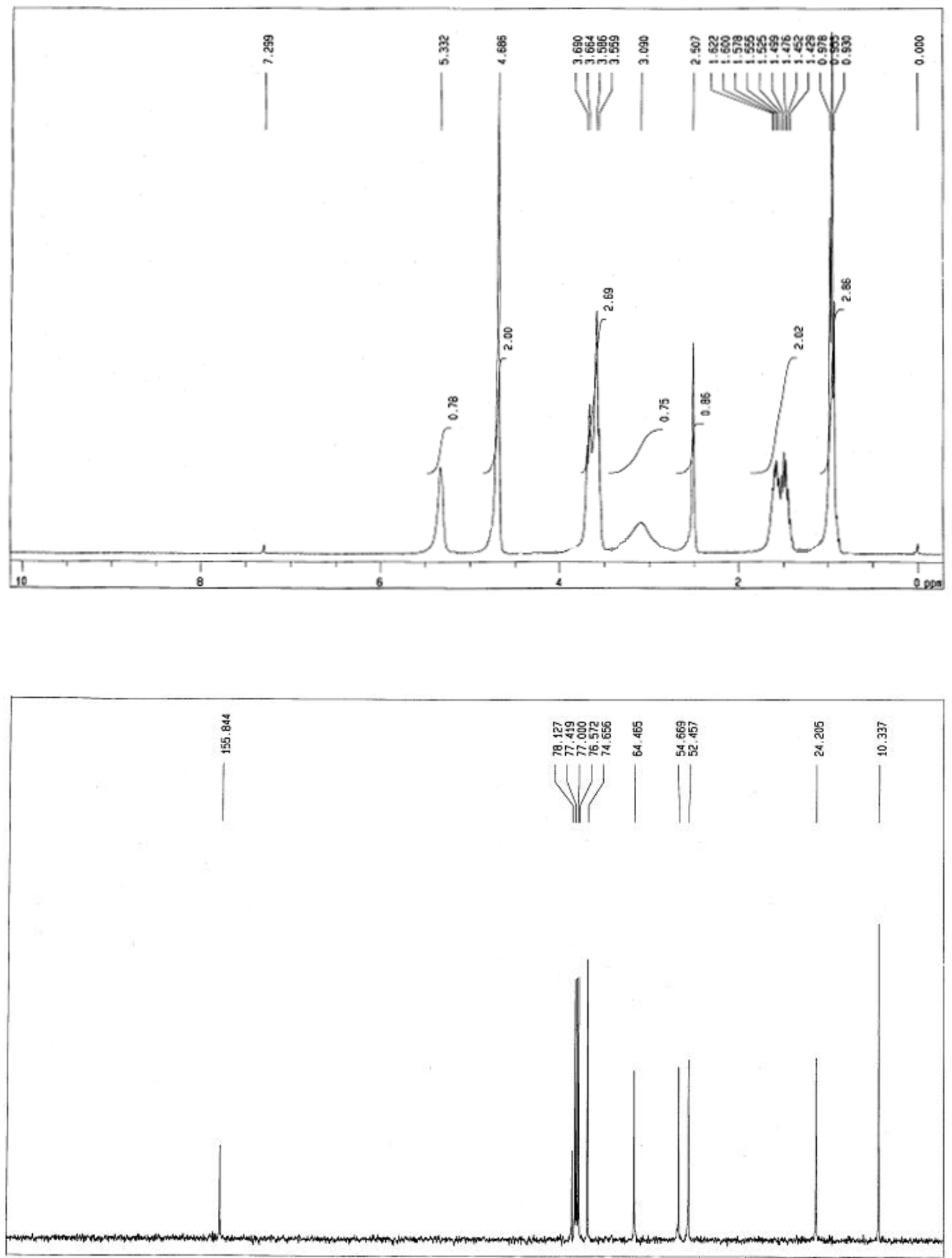
<smiles>C#CCOC(=O)NC(CO)CC(C)C</smiles>
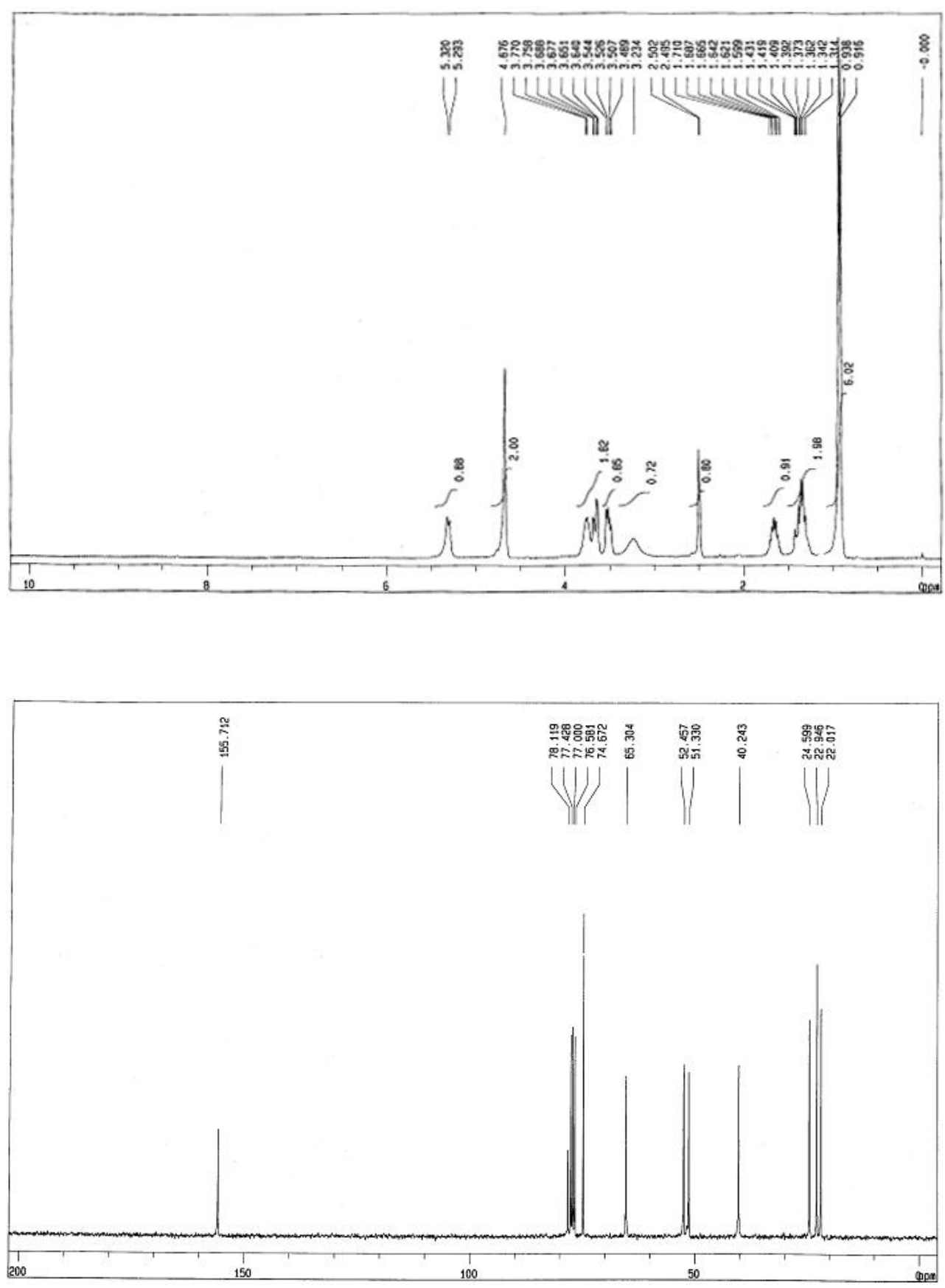
<smiles>C#CCOC(=O)NC(CO)Cc1ccccc1</smiles>
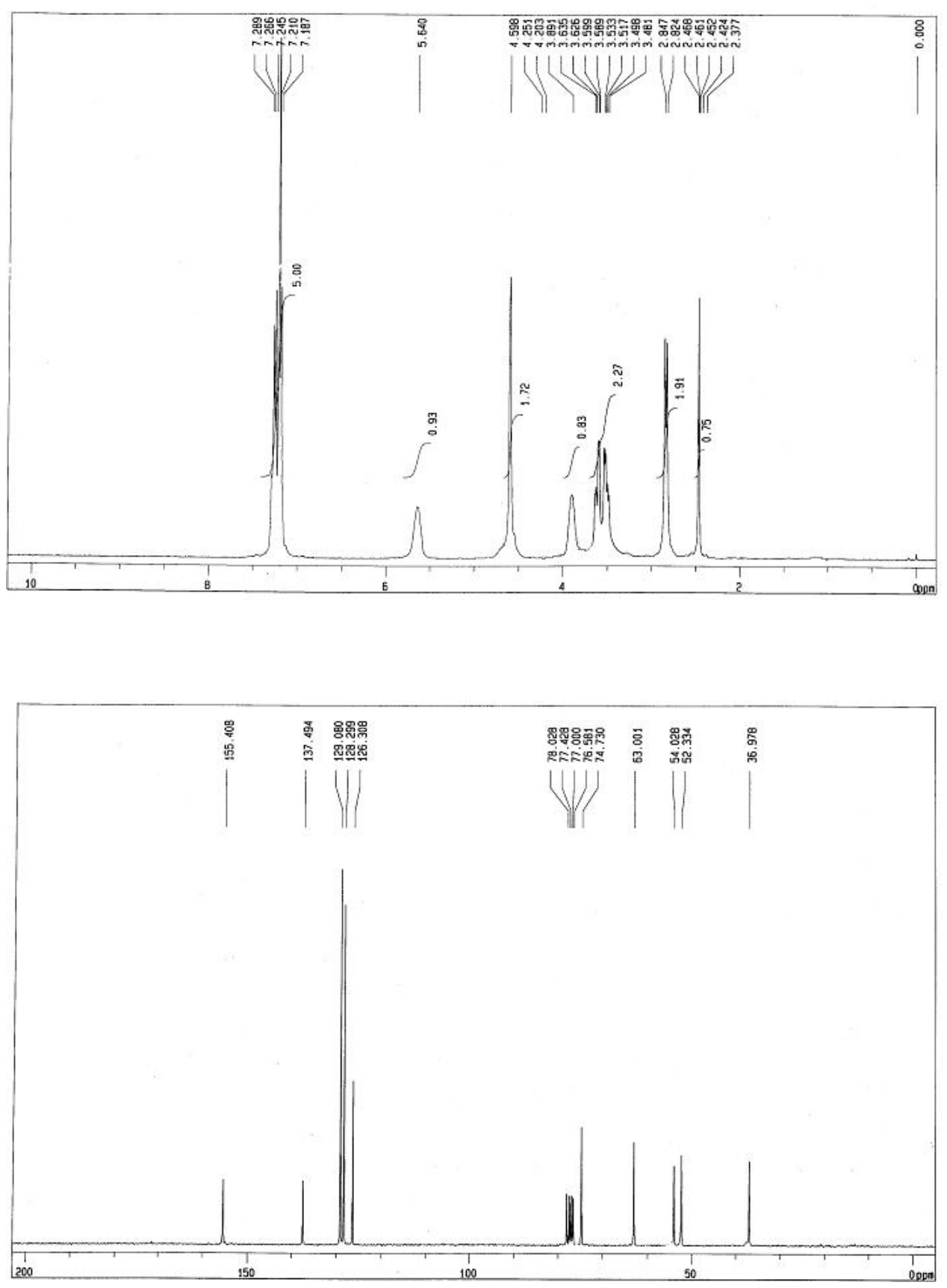
<smiles>C#CCOC(=O)NC(Cc1ccc(O)cc1)C(=O)OC</smiles>

\section{Compound 8d}
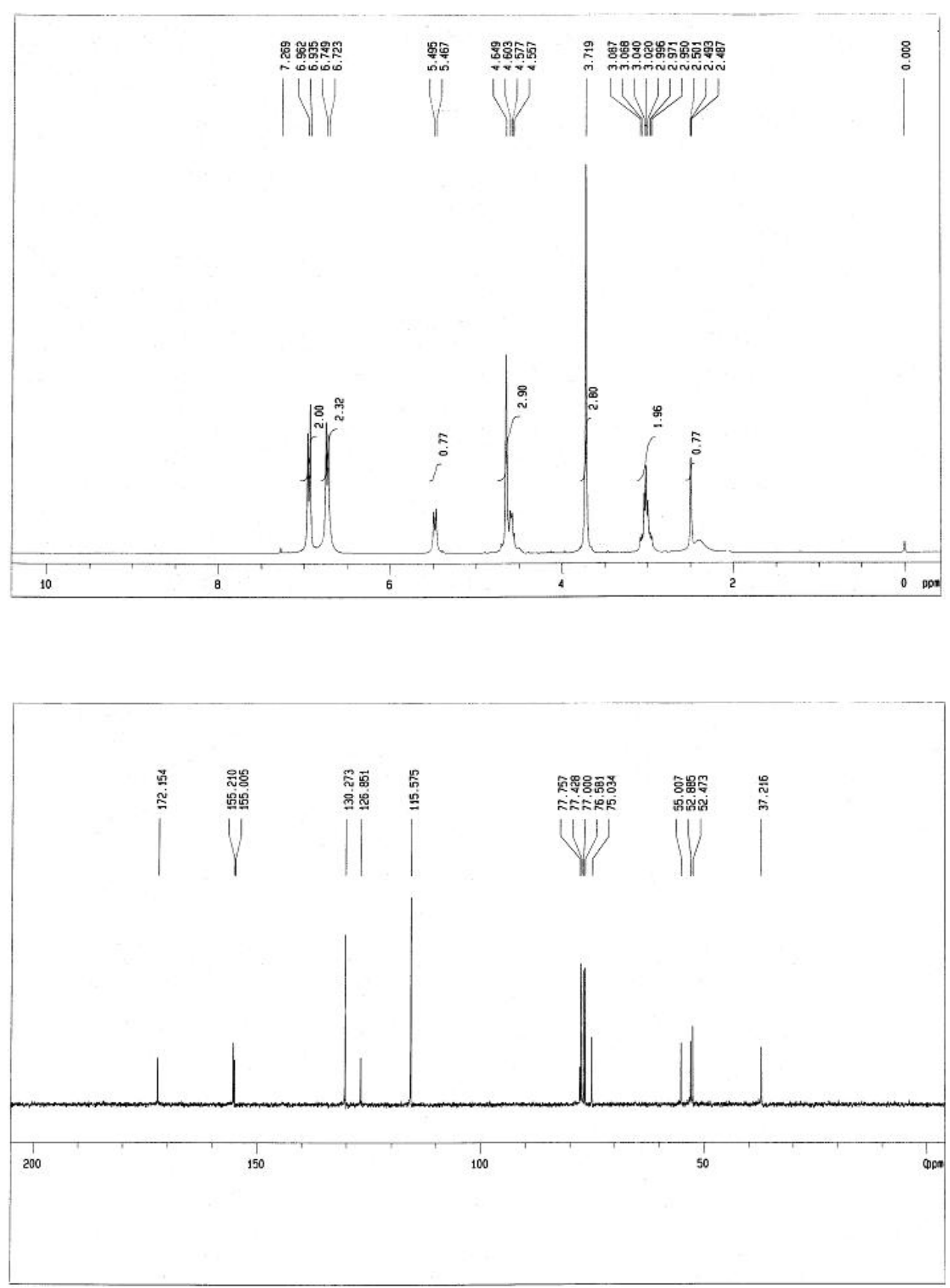
<smiles>C#CCOC(=O)NC(CO)C(=O)OC</smiles>
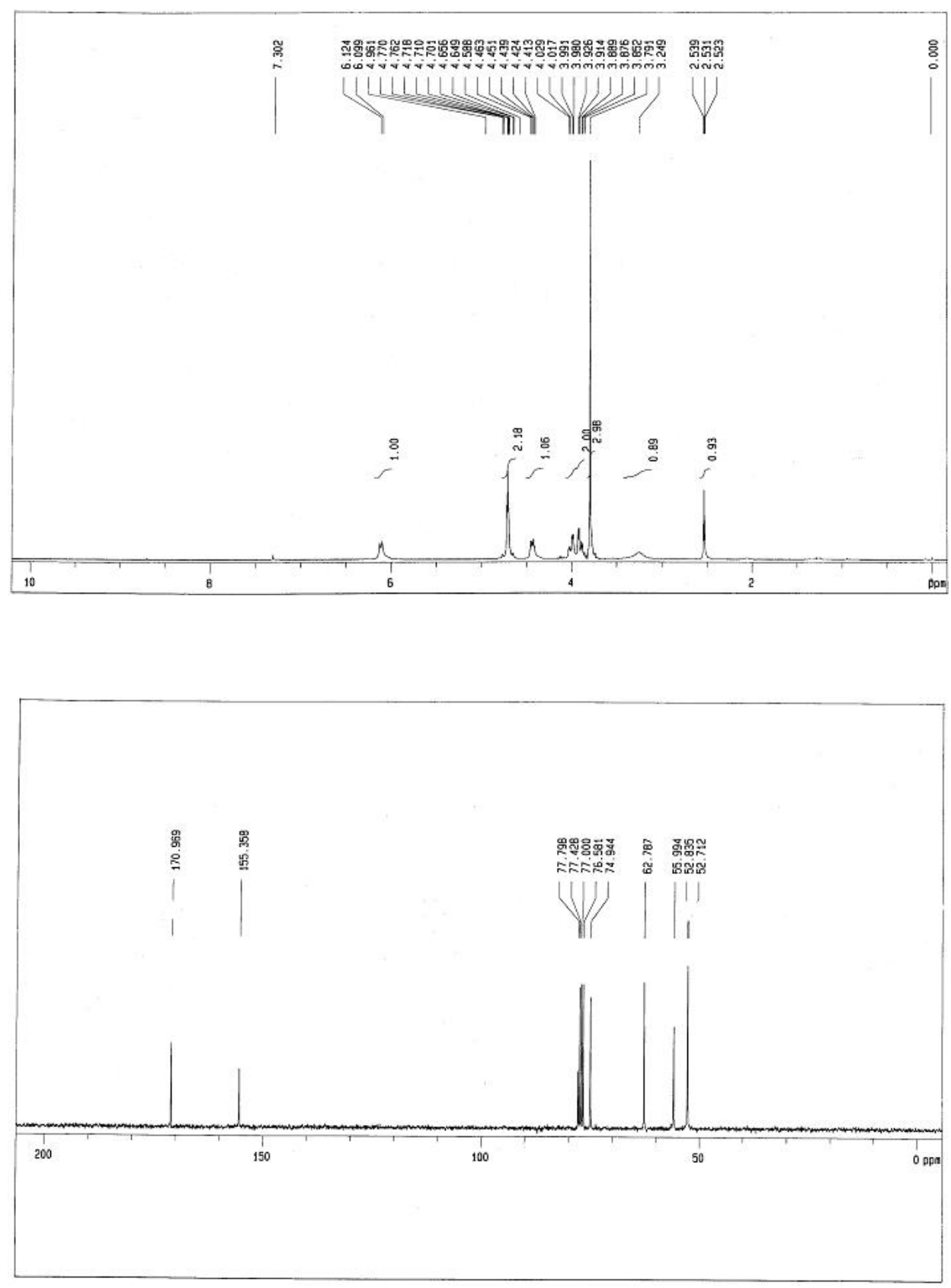
Compound 8f
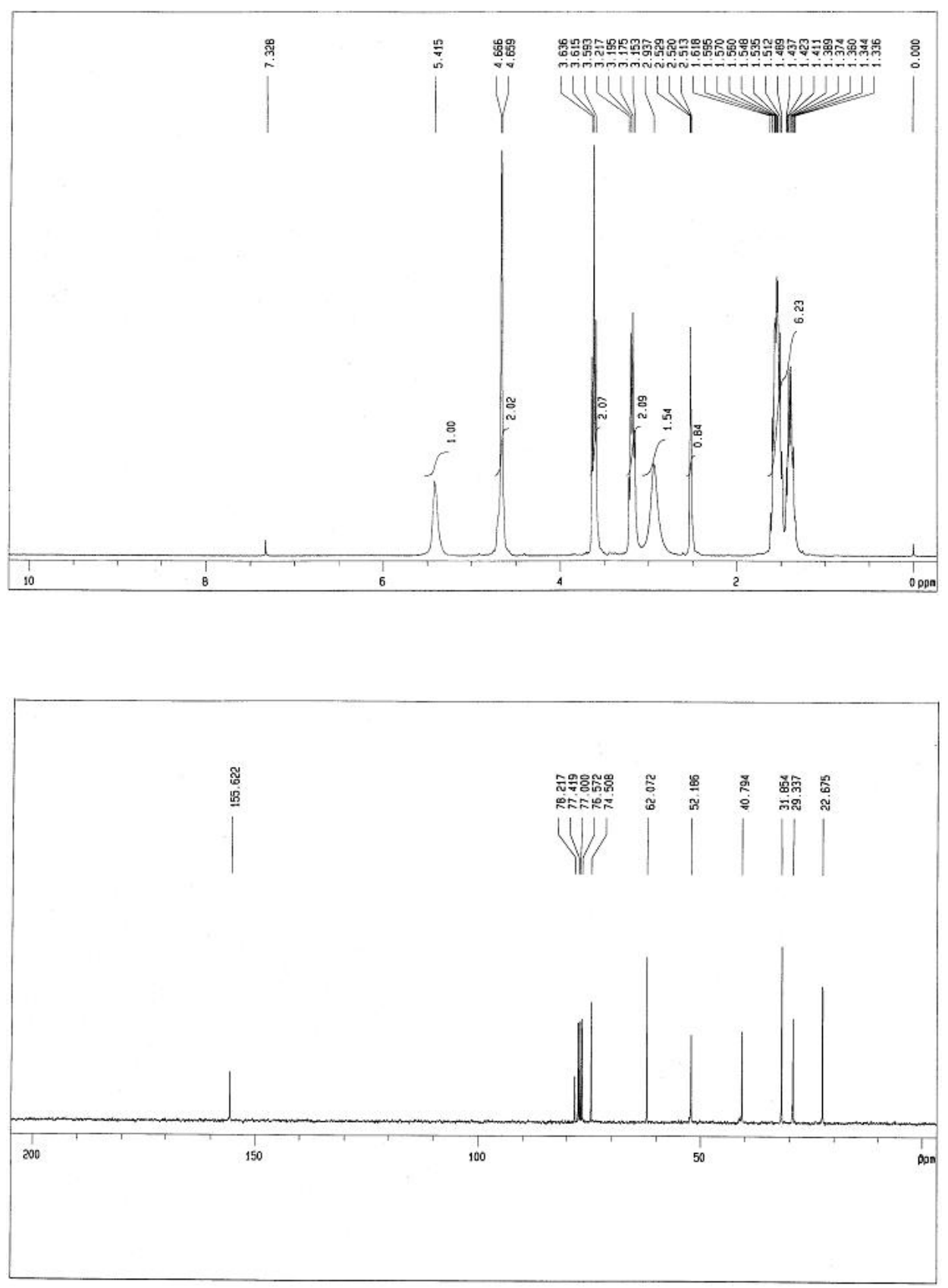
Compound 8g
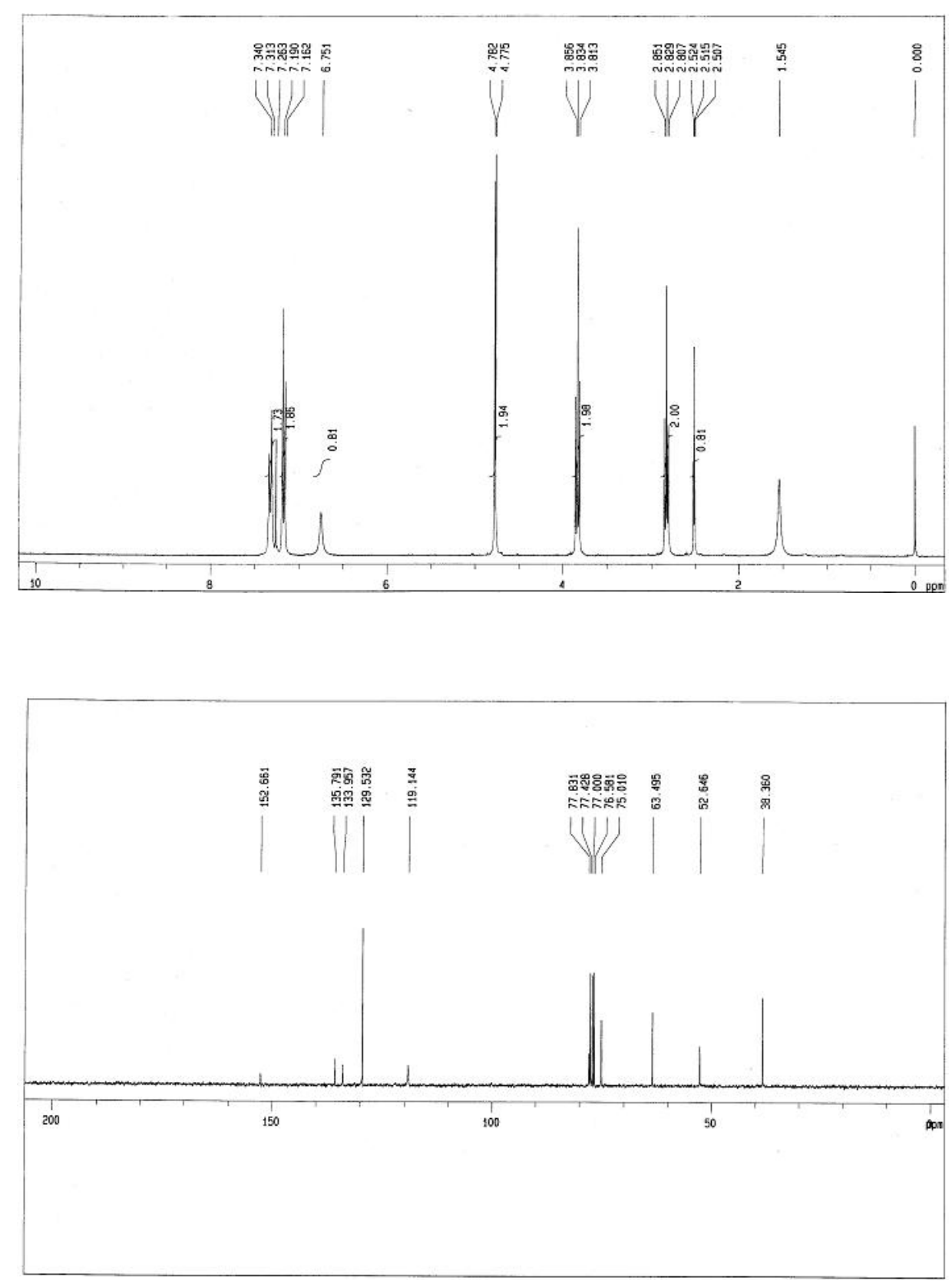
Compound 8h
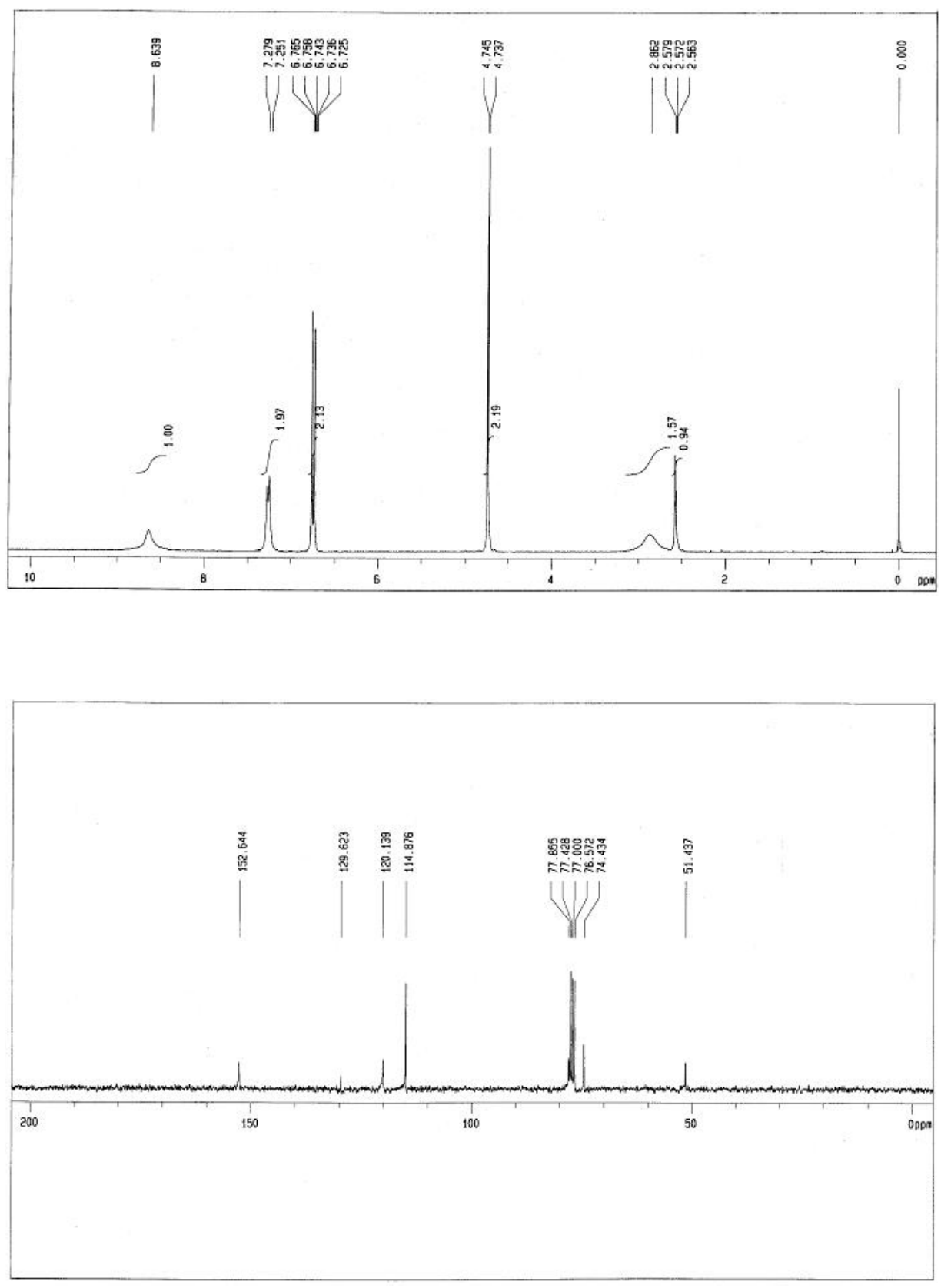